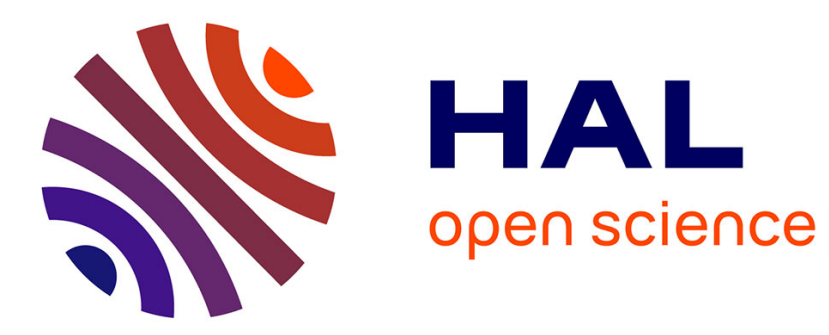

\title{
Design of reactionless linkages and robots equipped with balancing Assur groups
}

\author{
Sébastien Briot, Vigen Arakelian
}

\section{To cite this version:}

Sébastien Briot, Vigen Arakelian. Design of reactionless linkages and robots equipped with balancing Assur groups. Dan Zhang; Bin Wei. Dynamic Balancing of Mechanisms and Synthesizing of Parallel Robots, Springer, pp.33-68, 2016, 978-3-319-17682-6. 10.1007/978-3-319-17683-3_3 . hal-02166668

\section{HAL Id: hal-02166668 https://hal.science/hal-02166668}

Submitted on 27 Jun 2019

HAL is a multi-disciplinary open access archive for the deposit and dissemination of scientific research documents, whether they are published or not. The documents may come from teaching and research institutions in France or abroad, or from public or private research centers.
L'archive ouverte pluridisciplinaire HAL, est destinée au dépôt et à la diffusion de documents scientifiques de niveau recherche, publiés ou non, émanant des établissements d'enseignement et de recherche français ou étrangers, des laboratoires publics ou privés. 


\title{
Design of reactionless linkages and robots equipped with balancing Assur groups
}

\author{
Sébastien Briot and Vigen Arakelian
}

\begin{abstract}
In the present Chapter, we consider the shaking moment and shaking force balancing through the use of additional Assur groups mounted on the mechanism to be balanced. Two types of mechanisms are considered: (i) the in-line four-bar linkage and (ii) the planar parallel robots with prismatic pairs. For both types of mechanisms, the proposed solution allows the reduction (or even the cancellation in the case of the four-bar linkage) of the number of counter-rotations used for obtaining the shaking moment balancing, which decreases the design complexity and the inherent problems due to the use of counter-rotations (backlash, noise, vibrations, etc.). All theoretical developments are validated via simulations carried out using ADAMS software. The simulations show that the obtained mechanisms (both inline four-bar linkages and planar parallel robots) transmit no inertia loads to their surroundings, i.e. the sum of all ground bearing forces and their moments are eliminated.
\end{abstract}

\section{Introduction}

It is known that fast-moving machinery with rotating and reciprocating masses is a significant source of vibration excitation. The high-speed linkages can generate significant fluctuating forces with even small amounts of unbalance. Thus, a primary objective of the balancing is to cancel or reduce the variable dynamic loads transmitted to the frame and surrounding structures. The reduction of vibrations leads to the

S. Briot

Institut de Recherche en Communications et Cybernétique de Nantes (IRCCyN),

UMR CNRS 6597, Nantes - FRANCE, e-mail: Sebastien.Briot@irccyn.ec-nantes.fr

V. Arakelian

Institut National des Sciences Appliquées (INSA), Rennes - FRANCE

Institut de Recherche en Communications et Cybernétique de Nantes (IRCCyN),

UMR CNRS 6597, Nantes - FRANCE, e-mail: vigen.arakelian@insa-rennes.fr 
increased accuracy of manipulators [1], which is one of the positive consequences of the balancing. As was mentioned in [2], balancing brings other advantages such as a reduced cycle time [3], reduced noise, wear and fatigue [4], as well as improved ergonomics [5].

In general, two types of forces must be considered: the externally applied forces and the inertial forces. Inertial forces arise when links of a mechanism are subjected to large accelerations. The inertial force system acting on a given link can be represented as an inertia force acting on a line through the center of mass and an inertia torque about the center of mass. The determination of the inertial forces and torques is well known and it has been disclosed in various hand books [6]. With regard to the external forces, which are associated with the useful function that the mechanism is to perform, these are often smaller than inertia forces with a much lower variation. On the other hand, when formulating balancing conditions of a mechanism, it is necessary to recognize that, in many cases, external active forces applied to mechanism links constitute internal forces with respect to the mechanism as a whole. Thus, if all external active forces applied to the links of a mechanism are internal forces for the mechanism as whole, then the balance of the mechanism will be ensured under the fulfillment of inertia forces and inertia torque cancellation. Therefore, the balancing of shaking force and shaking moment due to the inertial forces of links acquires a specific importance. The quality of balancing of the moving masses has the influence not only on the level of vibrations but also on the resource, reliability and accuracy of mechanisms. Besides the mentioned negative effects, vibrations bring to the environments pollution and the loss of energy, and can also provoke various health issues. Consequently, the quality improvement of the mass balancing has not only technical, technological and economical aspects but also social.

Different approaches and solutions devoted to the shaking force and shaking moment balancing have been developed and documented for one-degree-of-freedom mechanisms [7-9]. Nowadays, a new field for balancing methods applications is the design of mechanical systems for fast manipulation [10], which is a typical problem in advanced robotics [11]. Here also we have similar problems relating to the cancelation or reduction of inertia forces. However, the mechanical systems with multi degrees of freedom lead to new solutions, such as the shaking force and shaking moment reduction by optimal motions of links, by adding flywheels with prescribed motions, or with the design of new self-balanced manipulators.

For all balancing method, the main challenge is the trade-off between the complexity of the balanced mechanism and the quality of balancing [9]. In the present Chapter, we propose a solution which allows the reduction of the balancing complexity by comparison with the usual approaches. The idea is to slightly modify the mechanism design by adding to it Assur groups, i.e. groups which dos not add any supplementary degree of freedom into the mechanism [12]. The use of such a solution is detailed in the following of the Chapter for the shaking force and shaking moment balancing of:

- the in-line four-bar linkage

- the planar parallel robots with prismatic pairs. 
For both types of mechanisms, the proposed solution allows the reduction (or even the cancellation in the case of the four-bar linkage) of the number of counterrotations used for obtaining the shaking moment balancing, which decreases the design complexity and the inherent problems due to the use of counter-rotations (backlash, noise, vibrations, etc.).

\section{Complete shaking force and shaking moment balancing of in-line four-bar linkages by adding a class-two RRR or RRP Assur group}

Many high-speed machines contain planar four-bar linkages and the problem of their mass balancing is of continuing interest to machine designers. The previous works on the balancing of planar four-bar linkages may be arranged in the following groups [6]:

1. Complete shaking force balancing [13-19]. In general, it is carried out by counterweights mounted on the movable links of the linkage. With regards to the several approaches employed for the redistribution of movables masses, the developed methods could be divided into three principal groups:

a. The method of "principal vectors" [13]; The aim of this approach was to study the balancing of the mechanism relative to each link and in the determination of those points on the links relative to which a static balance was obtained. These points were called "principal points". Then, from the condition of similarity of the vector loop of the principal points and the structural loop of the mechanism, the necessary conditions of balancing were derived.

b. The method of "static substitution of masses"; its aim was to statically substitute the mass of the coupler by concentrated masses, which are balanced thereafter together with the rotating links. Such an approach changes the problem of mechanism balancing into a simpler problem of balancing rotating links. This method was illustrated for four-bar linkage in [14-17].

c. The method of "linearly independent vectors" [18], in which the vector equation describing the position of the center of total mass of the mechanism is treated in conjunction with the closed equation of its kinematic chain. The result is an equation of static moments of moving link masses containing single linearly independent vectors. Thereafter following the conditions for balancing the mechanism by reducing the coefficients, which are time-dependent to zero.

It should be noted that the addition of a counterweighted pantograph device to the planar four-bar linkage has also been used for its complete shaking force balancing [19].

2. Complete shaking force and partial shaking moment balancing [20-29]. Two principal approaches may be distinguished: 
a. The shaking moment minimization of fully force balanced linkages [20-26], in which it is shown that the optimum conditions of partial moment balance can be obtained by certain link mass distribution ratios.

b. The minimization of the unbalance of shaking moment by transferring the rotation axis of the counterweight mounted on the input crank [27-29]. In the study [27], the first harmonic of the shaking moment is eliminated by attaching the required input link counterweight, not to the input shaft itself, but to a suitable offset one which rotates with the same angular velocity. This approach is original in that, while maintaining the force balance of the mechanism, it is possible to create an additional balancing moment, thereby reducing the shaking moment. This approach has been further developed in works $[28,29]$.

It should be noted that optimization algorithms are also widely used in partial balancing of four-bar linkages [30-33].

3. Complete shaking force and shaking moment balancing [9,34-43]. The first method of complete shaking force and shaking moment balancing was proposed in study [34], which was extended in [35]. In this approach, the mass of the connecting coupler is substituted dynamically by concentrated masses located at the coupler joints. Thus, the dynamic model of the coupler becomes a weightless link with two concentrated masses. This transforms the problem of four-bar linkage shaking force and shaking moment balancing into a problem of balancing rotating links carrying concentrated masses. The parallelogram structure has also been applied for complete shaking force and shaking moment balancing of fourbar linkages [36]. In the studies [37-41], the authors have proposed methods for complete shaking force and shaking moment balancing by counterweights with planetary gear trains. In [42] a toothed-belt transmission is used to rotate counterweights intended for shaking force balancing, which also allows shaking moment balancing. The disadvantage of these methods is the need for the connection of gears to the oscillating links. The oscillations of the links of the mechanism will create noise unless expensive anti-backlash gears are used.

Another solution using the copying properties of the pantograph was developed $[9,43]$, in which the gears driven by the coupler suffer no such sudden reversals so that this problem is almost eliminated. However, it should be noted that the use of the gears for the balancing of four-bar linkages is a drawback for the industrial applications and a fully shaking force and shaking moment balanced four-bar linkage without any gears is more appealing.

The shaking moment balancing of fully force balanced linkages using a prescribed input speed fluctuation was proposed in [44]. However, such balancing is complicated because it is necessary to use a special type of drive generator. Moreover, it cannot be used for balancing of linkages, which generate the prescribed motions of the output links.

In this section a solution is discussed, which allows the complete shaking force and shaking moment balancing of in-line four-bar linkages with constant input speed by adding a class-two Assur group, i.e. a group which does not add any supplemen- 
tary degree of freedom into the mechanism [12]. It should be noted that the balancing of the shaking moment without counter-rotations of three particular classes of four-bar linkages was discussed in the studies [45-49]. However, such a method cannot be extended to general four-bar linkages. In this section it is proposed to take advantages of the use of the properties of the four-bar linkage with prescribed geometric parameters [45-49] and to combine it with

1. the principle of the dynamic substitution of link mass by concentrated masses

2. and with the prescription of constant input speed.

It should be mentioned here that the suggested balancing approach can be efficiently applied on the cyclic high-speed machines executing motions in the steady-state regime when the input speed is constant $[50,51]$.

\subsection{Complete shaking force and shaking moment balancing by adding a class-two RRR Assur group}

\subsubsection{Theoretical background related to the balanced four-bar linkages with prescribed geometrical parameters}

Before considering the suggested balancing concept, let us recall basic notions concerning the balanced four-bar linkages with prescribed geometrical parameters.

In the paper of Berkof and Lowen [21], the angular momentum $H$ and the shaking moment $M^{s h}$, expressed at point $O$, of a force balanced in-line four-bar linkage (Fig. 1) were expressed as:

$$
H=\sum_{i=1}^{3} I_{i} \dot{\theta}_{i}, \quad M^{s h}=\frac{\mathrm{d} H}{\mathrm{~d} t}=\sum_{i=1}^{3} I_{i} \ddot{\theta}_{i}
$$

with

$$
\begin{gathered}
I_{i}=m_{i}\left(k_{i}^{2}+r_{i}^{2}+r_{i} l_{i}\right), \quad(i=1,3) \\
I_{2}=m_{2}\left(k_{2}^{2}+r_{2}^{2}-r_{2} l_{2}\right)
\end{gathered}
$$

where $r_{i}$ is the length of vector $\mathbf{r}_{i}$ which connects the pivot $P_{i}$ to the centre of mass $S_{i}$ of link $i, l_{i}$ is the length of vector $\mathbf{l}_{i}$ which connects the proximal revolute joint $P_{i}$ to the distal joint on the same link, and $k_{i}$ is the radius of gyration with respect to the centre of mass of link $i, m_{i}$ is the mass of link $i$. Moreover, $\theta_{i}$ is the angular position of link $i$ with respect to the $\mathbf{x}$-axis.

With regard to the shaking force balancing, the following expressions were obtained:

$$
\begin{gathered}
m_{1} r_{1}=m_{2} l_{1} \frac{l_{2}-r_{2}}{l_{2}} \\
m_{3} r_{3}=m_{2} r_{2} \frac{l_{3}}{l_{2}}
\end{gathered}
$$




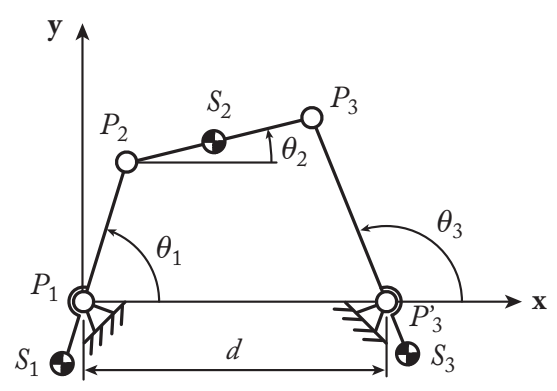

Fig. 1 A general in-line four-bar mechanism.

In the works $[47,52]$, it has been shown that it is possible to cancel the shaking moment of a four-bar mechanism by associating mentioned geometric constraints with an optimal distribution of masses. Three kinds of shaking force and shaking moment balanced four-bar mechanisms were found, which are shown in Fig. 2.

In order to illustrate the shaking moment balancing, let us consider the mechanism shown in Fig. 2(b). The geometrical constraints of this mechanism are the following:

$$
\begin{aligned}
& l_{1}=l_{3} \\
& d=l_{2}
\end{aligned}
$$

where $d$ is the length of the base which is the distance between the two fixed joints on the base.

This leads to the following kinematic relationships:

$$
\dot{\theta}_{1}-\dot{\theta}_{2}+\dot{\theta}_{3}=0
$$

Thus, from expressions (1) and (8), it is easy to see that the shaking moment will be cancelled if $I_{1}=I_{2}=I_{3}$ (see Eqs. (2) and (3)). For this purpose the following relationships must be established:

$$
\begin{aligned}
& k_{2}^{2}=\frac{m_{2}\left(l_{2} r_{2}-r_{2}^{2}\right)-I_{1}}{m_{2}} \\
& k_{3}^{2}=\frac{-m_{3}\left(l_{3} r_{3}+r_{3}^{2}\right)+I_{1}}{m_{3}}
\end{aligned}
$$

where

$$
I_{1}=m_{1}\left(k_{1}^{2}+r_{1}^{2}+r_{1} l_{1}\right)
$$

It should be noted that similar results have been obtained for the mechanisms shown in Figs. 2(a) and 2(c). 


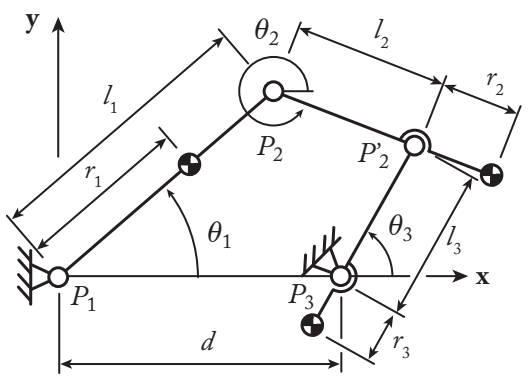

(a) Case I: $l_{1}=d$ and $l_{2}=l_{3}$

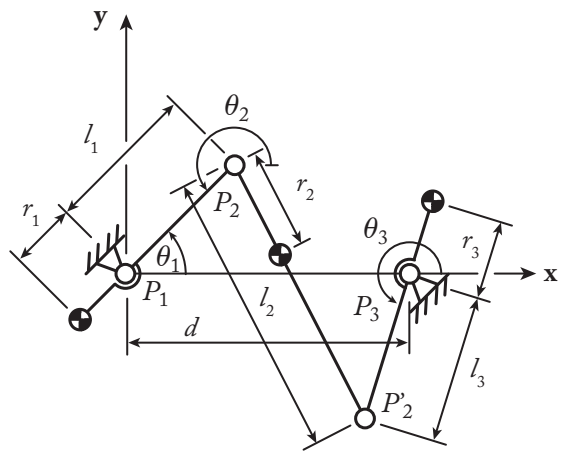

(b) Case II: $l_{2}=d$ and $l_{1}=l_{3}$

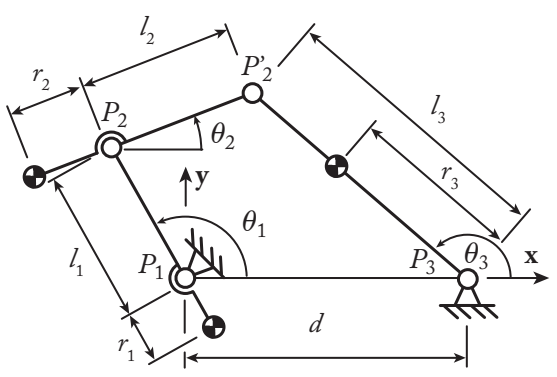

(c) Case III: $l_{3}=d$ and $l_{1}=l_{2}$

Fig. 2 The three kinds of shaking force and shaking moment balanced four-bar mechanisms.

Statement of the problem: the aim of the suggested balancing approach consists of adding a two-link kinematic chain with prescribed geometrical parameters to an inline four-bar linkage with arbitrary geometrical parameters. It is important to note that the added structure must be an Assur group, i.e. a group which does not add any supplementary degree of freedom into the mechanism [12]. This allows for the modification of the mass redistribution of the obtained six-bar mechanism without perturbation of the kinematic properties of the initial four-bar linkage. We would like to state that this technique allows for the complete shaking force and shaking moment balancing without counter-rotating masses.

Now let us consider the shaking force and shaking moment balancing of an inline four-bar mechanism using class-two Assur groups with $R R R$ kinematic chain. 


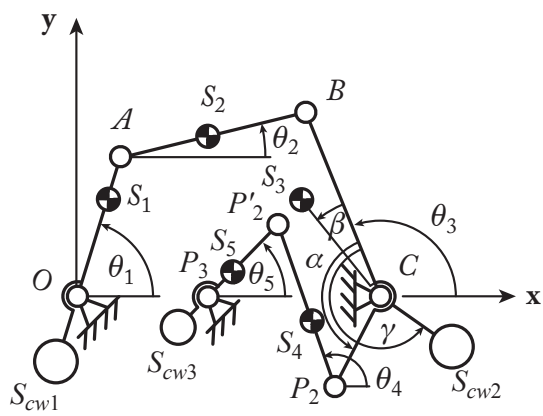

Fig. 3 The balanced mechanism with the class-two $R R R$ Assur group.

\subsubsection{Shaking force balancing}

Figure 3 shows an in-line four-bar linkage with the added class-two $R R R$ Assur group. Let us denote the following vectors as: $\mathbf{l}_{1}=\mathbf{d}_{O A}, \mathbf{l}_{2}=\mathbf{d}_{A B}, \mathbf{l}_{3}=\mathbf{d}_{C B}, \mathbf{l}_{3}^{\prime}=$ $\mathbf{d}_{C P_{2}}, \mathbf{l}_{4}=\mathbf{d}_{P_{2} P_{2}^{\prime}}, \mathbf{l}_{5}=\mathbf{d}_{P_{3} P_{2}^{\prime}}, \mathbf{r}_{1}=\mathbf{d}_{O S_{1}}, \mathbf{r}_{2}=\mathbf{d}_{A S_{2}}, \mathbf{r}_{3}=\mathbf{d}_{C S_{3}}, \mathbf{r}_{4}=\mathbf{d}_{P_{2} S_{4}}, \mathbf{r}_{5}=\mathbf{d}_{P_{3} S_{5}}$, $\mathbf{r}_{c w_{1}}=\mathbf{d}_{O S_{c w_{1}}}, \mathbf{r}_{c w 2}=\mathbf{d}_{C S_{c w_{2}}}, \mathbf{r}_{c w 3}=\mathbf{d}_{P_{3} S_{c w_{3}}}$.

The added class-two RRR Assur group has the above mentioned properties, i.e. it is designed such as:

$$
\begin{gathered}
l_{3}^{\prime}=l_{5} \\
e=l_{4}
\end{gathered}
$$

where $l_{i}(i=1 \ldots 5)$ is the norm of vector $\mathbf{l}_{i}$ and $e$ the distance between $C$ and $P_{3}$.

As is shown in Fig. 3, the Assur group $P_{2} P_{2}^{\prime} P_{3}$ is attached to the initial linkage $O A B C$ in such a way that it forms a four-bar linkage with link $B C$, as discussed in Section 2.1.1.

Let us now derive the expression of the shaking force $\mathbf{f}^{\text {sh }}$ of the obtained mechanism:

$$
\mathbf{f}^{s h}=\sum_{i=1}^{5} m_{i} \ddot{\mathbf{d}}_{S_{i}}
$$

where $\ddot{\mathbf{d}}_{S_{i}}$ is the translational acceleration of the centre of mass $S_{i}$ and $m_{i}$ the mass of the link $i$. Developing and simplifying, one obtains:

$$
\begin{aligned}
\mathbf{f}^{s h}= & \left(\frac{m_{1} r_{1}}{l_{1}}+m_{2} \frac{l_{2}-r_{2}}{l_{2}}\right) \ddot{\mathbf{d}}_{A}+m_{2} \frac{r_{2}}{l_{2}} \ddot{\mathbf{d}}_{B}+m_{3} \ddot{\mathbf{d}}_{S_{3}} \\
& +m_{4} \frac{l_{4}-r_{4}}{l_{4}} \ddot{\mathbf{d}}_{P_{2}}+\left(\frac{m_{4} r_{4}}{l_{4}}+\frac{m_{5} r_{5}}{l_{5}}\right) \ddot{\mathbf{d}}_{P_{2}^{\prime}}
\end{aligned}
$$

where $r_{i}$ is the algebraic values of the norm of vectors $\mathbf{r}_{i}$, and $\ddot{\mathbf{d}}_{A}, \ddot{\mathbf{d}}_{B}, \ddot{\mathbf{d}}_{S_{3}}, \ddot{\mathbf{d}}_{P_{2}}$ and $\ddot{\mathbf{d}}_{P_{2}^{\prime}}$ represent the acceleration of points $A, B, S_{3}, P_{2}$ and $P_{2}^{\prime}$ respectively. Their expressions are: 


$$
\begin{gathered}
\ddot{\mathbf{d}}_{A}=l_{1}\left(\ddot{\theta}_{1}\left[\begin{array}{c}
-\sin \theta_{1} \\
\cos \theta_{1}
\end{array}\right]-\dot{\theta}_{1}^{2}\left[\begin{array}{c}
\cos \theta_{1} \\
\sin \theta_{1}
\end{array}\right]\right) \\
\ddot{\mathbf{d}}_{3}=l_{3}\left(\ddot{\theta}_{3}\left[\begin{array}{c}
-\sin \theta_{3} \\
\cos \theta_{3}
\end{array}\right]-\dot{\theta}_{3}^{2}\left[\begin{array}{c}
\cos \theta_{3} \\
\sin \theta_{3}
\end{array}\right]\right) \\
\ddot{\mathbf{d}}_{S_{3}}=r_{3}\left(\ddot{\theta}_{3}\left[\begin{array}{c}
-\sin \left(\theta_{3}+\beta\right) \\
\cos \left(\theta_{3}+\beta\right)
\end{array}\right]-\dot{\theta}_{3}^{2}\left[\begin{array}{c}
\cos \left(\theta_{3}+\beta\right) \\
\sin \left(\theta_{3}+\beta\right)
\end{array}\right]\right) \\
\ddot{\mathbf{d}}_{P_{2}}=l_{3}^{\prime}\left(\ddot{\theta}_{3}\left[\begin{array}{c}
-\sin \left(\theta_{3}+\alpha\right) \\
\cos \left(\theta_{3}+\alpha\right)
\end{array}\right]-\dot{\theta}_{3}^{2}\left[\begin{array}{c}
\cos \left(\theta_{3}+\alpha\right) \\
\sin \left(\theta_{3}+\alpha\right)
\end{array}\right]\right) \\
\ddot{\mathbf{d}}_{P_{2}^{\prime}}=l_{5}\left(\ddot{\theta}_{5}\left[\begin{array}{c}
-\sin \theta_{5} \\
\cos \theta_{5}
\end{array}\right]-\dot{\theta}_{5}^{2}\left[\begin{array}{c}
\cos \theta_{5} \\
\sin \theta_{5}
\end{array}\right]\right)
\end{gathered}
$$

in which the angles $\theta_{i}(i=1 \ldots 5)$ are defined in Fig. 3.

The shaking force $\mathbf{f}^{\text {sh }}$ may be cancelled through the addition of three counterweights positioned at points $S_{c w_{i}}$ (Fig. 3), with masses $m_{c w_{i}}(i=1,2,3)$. With such counterweights, the expression of the shaking force becomes:

$$
\mathbf{f}^{s h^{*}}=\mathbf{f}^{s h}+m_{c w_{1}} \frac{r_{c w_{1}}}{l_{1}} \ddot{\mathbf{d}}_{A}+m_{c w_{2}} \ddot{\mathbf{d}}_{S_{c w_{2}}}+m_{c w_{3}} \frac{r_{c w_{3}}}{l_{5}} \ddot{\mathbf{d}}_{P_{2}^{\prime}}
$$

where $r_{c w_{i}}$ is the algebraic values of the norm of vectors $\mathbf{r}_{c w_{i}}$ and

$$
\ddot{\mathbf{d}}_{S_{c w_{2}}}=r_{c w_{2}}\left(\ddot{\theta}_{3}\left[\begin{array}{c}
-\sin \left(\theta_{3}+\gamma\right) \\
\cos \left(\theta_{3}+\gamma\right)
\end{array}\right]-\dot{\theta}_{3}^{2}\left[\begin{array}{c}
\cos \left(\theta_{3}+\gamma\right) \\
\sin \left(\theta_{3}+\gamma\right)
\end{array}\right]\right)
$$

Thus the shaking force is cancelled if the distribution of the masses is as follows:

$$
\begin{gathered}
m_{c w_{1}}=-\frac{l_{1}}{r_{c w_{1}}}\left(m_{1} \frac{r_{1}}{l_{1}}+m_{2} \frac{l_{2}-r_{2}}{l_{2}}\right) \\
\tan \gamma=\frac{m_{3} r_{3} \sin \beta+m_{4}\left(1-r_{4} / l_{4}\right) l_{3}^{\prime} \sin \alpha}{m_{2} l_{3} r_{2} / l_{2}+m_{3} r_{3} \cos \beta+m_{4}\left(1-r_{4} / l_{4}\right) l_{3}^{\prime} \sin \alpha} \\
m_{c w_{2}}^{2}=\frac{\left(m_{3} r_{3} \sin \beta+m_{4}\left(1-r_{4} / l_{4}\right) l_{3}^{\prime} \sin \alpha\right)^{2}}{r_{c w_{2}}^{2}} \\
+\frac{\left(m_{2} l_{3} r_{2} / l_{2}+m_{3} r_{3} \cos \beta+m_{4}\left(1-r_{4} / l_{4}\right) l_{3}^{\prime} \cos \alpha\right)^{2}}{r_{c w_{2}}^{2}} \\
m_{c w_{3}}=-\frac{l_{5}}{r_{c w_{3}}}\left(m_{4} \frac{r_{4}}{l_{4}}+m_{5} \frac{r_{5}}{l_{5}}\right)
\end{gathered}
$$

\subsubsection{Shaking moment balancing}

Let us now derive the expression of the shaking moment $M^{\text {sh }}$, expressed at point $O$, of such a mechanism: 


$$
M^{s h}=\sum_{i=1}^{5} m_{i}\left(x_{S_{i}} \ddot{y}_{S_{i}}-y_{S_{i}} \ddot{x}_{S_{i}}+k_{i}^{2} \ddot{\theta}_{i}\right)+\sum_{i=1}^{3} m_{c w_{i}}\left(x_{S_{c w_{i}}} \ddot{y}_{S_{c w_{i}}}-y_{S_{c w_{i}}} \ddot{x}_{S_{c w_{i}}}\right)
$$

where $x_{S_{i}}, y_{S_{i}}, \ddot{x}_{S_{i}}$ and $\ddot{y}_{S_{i}}$ are the position and accelerations along $\mathbf{x}$ and $\mathbf{y}$ axes of points $S_{i}$, respectively, and $x_{S_{c w_{i}}}, y_{S_{c w_{i}}}, \ddot{x}_{S_{c w_{i}}}$ and $\ddot{y}_{S_{c w_{i}}}$ are the position and accelerations along $\mathbf{x}$ and $\mathbf{y}$ axes of points $S_{c w_{i}}$, respectively, $k_{i}$ is the radius of gyration of link $i$.

Now, let us consider that link 2 is a physical pendulum ${ }^{1}$ [35], i.e. it can be replaced dynamically by two point masses located at joint centres $A$ and $B$. This implies that:

$$
k_{2}^{2}=r_{2}\left(l_{2}-r_{2}\right)
$$

Then, considering that the input speed is constant, i.e. $\ddot{\theta}_{1}=0$ and taking into account (28), one can simplify (27) as:

$$
M^{s h}=\sum_{i=3}^{5} I_{i} \ddot{\theta}_{i}
$$

where

$$
\begin{gathered}
I_{3}=m_{3}\left(k_{3}^{2}+r_{3}^{2}\right)+m_{2} \frac{r_{2}}{l_{2}} l_{3}^{2}+m_{c w_{2}} r_{c w_{2}}^{2}+m_{4} \frac{l_{4}-r_{4}}{l_{4}} l_{3}^{\prime 2} \\
I_{4}=m_{4}\left(k_{4}^{2}+r_{4}^{2}-r_{4} l_{4}\right) \\
I_{5}=m_{5}\left(k_{5}^{2}+r_{5}^{2}-r_{c w_{3}} r_{5}\right)+m_{4} \frac{r_{4}}{l_{4}} l_{5}\left(l_{5}-r_{c w_{3}}\right)
\end{gathered}
$$

Thus, this new six-bar mechanism has the same shaking moment as the four-bar mechanism composed of links $P_{1} P_{2}, P_{2} P_{2}^{\prime}$ and $P_{2}^{\prime} P_{3}$. Therefore, the initial four-bar linkage balancing problem is transformed in the balancing of the four-bar linkage formed by the added Assur group. Note that the latter has specific geometry and its balancing conditions have been examined in Section 2.1.1.

Applying these results to the considered mechanism, we obtain:

$$
\begin{gathered}
k_{4}^{2}=\frac{-I_{3}-r_{4}^{2}+r_{4} l_{4}}{m_{4}} \\
k_{5}^{2}=\frac{m_{5}\left(-r_{5}^{2}+r_{c w_{3}} r_{5}\right)+m_{4} r_{4} / l_{4} l_{5}\left(l_{5}-r_{c w_{3}}\right)+I_{3}}{m_{5}}
\end{gathered}
$$

The substitution of (33)-(34) into (29) leads to:

$$
M^{s h}=I_{3}\left(\ddot{\theta}_{3}-\ddot{\theta}_{4}+\ddot{\theta}_{5}\right)
$$

Taking into account relations (6) and (6), we have

\footnotetext{
${ }^{1}$ A "physical pendulum" is a link which has such a distribution of masses that it allows the dynamic substitution of link's mass and inertia by two concentrated masses.
} 


$$
\ddot{\theta}_{3}-\ddot{\theta}_{4}+\ddot{\theta}_{5}=0
$$

and consequently

$$
M^{s h}=0
$$

The proposed balancing technique has been illustrated using the four-bar linkage shown in Fig. 2(b). However, it can also be achieved via the mechanism of Fig. 2(a) or Fig. 2(c).

\subsection{Complete shaking force and shaking moment balancing by adding a class-two RRP Assur group}

\subsubsection{Shaking force balancing}

The second solution, which is proposed for the cancellation of the shaking moment of a four-bar linkage is carried out by adding a class-two RRP Assur group (Fig. 4). Let us denote the following vectors as: $\mathbf{l}_{1}=\mathbf{d}_{O A}, \mathbf{l}_{2}=\mathbf{d}_{A B}, \mathbf{l}_{3}=\mathbf{d}_{C B}, \mathbf{l}_{3}^{\prime}=\mathbf{d}_{C D}$, $\mathbf{l}_{4}=\mathbf{d}_{D E}, \mathbf{r}_{1}=\mathbf{d}_{O S_{1}}, \mathbf{r}_{2}=\mathbf{d}_{A S_{2}}, \mathbf{r}_{3}=\mathbf{d}_{C S_{3}}, \mathbf{r}_{4}=\mathbf{d}_{D S_{4}}, \mathbf{r}_{5}=\mathbf{d}_{E S_{5}}, \mathbf{r}_{c w_{1}}=\mathbf{d}_{O S_{C w_{1}}}$, $\mathbf{r}_{c w 2}=\mathbf{d}_{C S_{c w_{2}}}, \mathbf{r}_{c w 3}=\mathbf{d}_{D S_{c w_{3}}}$.

In this case, the lengths of added links are the following:

$$
l_{3}^{\prime}=l_{4}
$$

Thus, the new part created by added links is a Scott-Russell mechanism [53], which is attached to the initial linkage at an angle of $\alpha$.

The relations between the link accelerations are the following for the ScottRussell mechanism:

$$
\dot{\theta}_{3}=-\dot{\theta}_{4}
$$

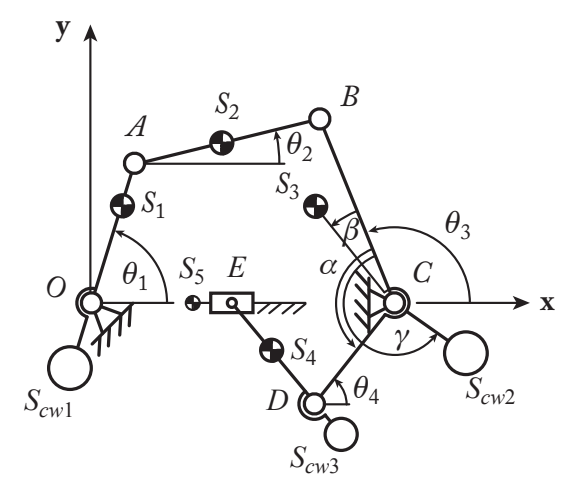

Fig. 4 The balanced mechanism with the class-two RRP Assur group. 
Let us now derive the expression of the shaking force $\mathbf{f}^{\text {sh }}$ of such a mechanism:

$$
\mathbf{f}^{s h}=\sum_{i=1}^{5} m_{i} \ddot{\mathbf{d}}_{S_{i}}
$$

Developing and simplifying, one obtains:

$$
\begin{aligned}
\mathbf{f}^{s h}= & \left(\frac{m_{1} r_{1}}{l_{1}}+m_{2} \frac{l_{2}-r_{2}}{l_{2}}\right) \ddot{\mathbf{d}}_{A}+m_{2} \frac{r_{2}}{l_{2}} \ddot{\mathbf{d}}_{B}+m_{3} \ddot{\mathbf{d}}_{S_{3}} \\
& +\left(m_{4}+m_{5}\right) \ddot{\mathbf{d}}_{D}+\left(m_{4} \frac{r_{4}}{l_{4}}+m_{5}\right) \ddot{\mathbf{d}}
\end{aligned}
$$

where $r_{i}$ and $l_{i}$ are the algebraic values of the norm of vectors $\mathbf{r}_{i}$, and $\mathbf{l}_{i}$, respectively,

$$
\begin{gathered}
\ddot{\mathbf{d}}_{S_{3}}=r_{3}\left(\ddot{\theta}_{3}\left[\begin{array}{c}
-\sin \left(\theta_{3}+\beta\right) \\
\cos \left(\theta_{3}+\beta\right)
\end{array}\right]-\dot{\theta}_{3}^{2}\left[\begin{array}{c}
\cos \left(\theta_{3}+\beta\right) \\
\sin \left(\theta_{3}+\beta\right)
\end{array}\right]\right) \\
\ddot{\mathbf{d}}=l_{5}\left(\ddot{\theta}_{4}\left[\begin{array}{c}
-\sin \theta_{4} \\
\cos \theta_{4}
\end{array}\right]-\dot{\theta}_{4}^{2}\left[\begin{array}{c}
\cos \theta_{4} \\
\sin \theta_{4}
\end{array}\right]\right) \\
\ddot{\mathbf{d}}_{D}=l_{3}^{\prime}\left(\ddot{\theta}_{3}\left[\begin{array}{c}
-\sin \left(\theta_{3}+\alpha\right) \\
\cos \left(\theta_{3}+\alpha\right)
\end{array}\right]-\dot{\theta}_{3}^{2}\left[\begin{array}{c}
\cos \left(\theta_{3}+\alpha\right) \\
\sin \left(\theta_{3}+\alpha\right)
\end{array}\right]\right)
\end{gathered}
$$

in which the angles $\theta_{i}(i=1 \ldots 4)$ are defined in Fig. 4.

The shaking force $\mathbf{f}^{\text {sh }}$ may be cancelled through the addition of three counterweights positioned at points $S_{c w_{i}}$ (Fig. 4), with masses $m_{c w_{i}}(i=1,2,3)$. With such counterweights, the expression of the shaking force becomes:

$$
\mathbf{f}^{s h^{*}}=\mathbf{f}^{s h}+m_{c w_{1}} \frac{r_{c w_{1}}}{l_{1}} \ddot{\mathbf{d}}_{A}+m_{c w_{2}} \ddot{\mathbf{d}}_{S_{c w_{2}}}+m_{c w_{3}} \ddot{\mathbf{d}}_{D}+m_{c w_{3}} \frac{r_{c w_{3}}}{l_{4}} \ddot{\mathbf{d}}
$$

where $r_{c w_{i}}$ is the algebraic values of the norm of vectors $\mathbf{r}_{c w_{i}}$ and

$$
\ddot{\mathbf{d}}_{S_{c w_{2}}}=r_{c w_{2}}\left(\ddot{\theta}_{3}\left[\begin{array}{c}
-\sin \left(\theta_{3}+\gamma\right) \\
\cos \left(\theta_{3}+\gamma\right)
\end{array}\right]-\dot{\theta}_{3}^{2}\left[\begin{array}{c}
\cos \left(\theta_{3}+\gamma\right) \\
\sin \left(\theta_{3}+\gamma\right)
\end{array}\right]\right)
$$

Thus the shaking force is cancelled if the distribution of the masses is as follows:

$$
\begin{gathered}
m_{c w_{1}}=-\frac{l_{1}}{r_{c w_{1}}}\left(m_{1} \frac{r_{1}}{l_{1}}+m_{2} \frac{l_{2}-r_{2}}{l_{2}}\right) \\
\tan \gamma=\frac{m_{3} r_{3} \sin \beta+\left(m_{4}+m_{5}\right) l_{3}^{\prime} \sin \alpha}{m_{2} l_{3} r_{2} / l_{2}+m_{3} r_{3} \cos \beta+\left(m_{4}+m_{5}\right) l_{3}^{\prime} \sin \alpha}
\end{gathered}
$$




$$
\begin{aligned}
& m_{c w_{2}}^{2}= \frac{\left(m_{3} r_{3} \sin \beta+\left(m_{4}+m_{5}\right) l_{3}^{\prime} \sin \alpha\right)^{2}}{r_{c w_{2}}^{2}} \\
&+ \frac{\left(m_{2} l_{3} r_{2} / l_{2}+m_{3} r_{3} \cos \beta+\left(m_{4}+m_{5}\right) l_{3}^{\prime} \cos \alpha\right)^{2}}{r_{c w_{2}}^{2}} \\
& m_{c w_{3}}=-\frac{l_{4}}{r_{c w_{3}}}\left(m_{4} \frac{r_{4}}{l_{4}}+m_{5}\right)
\end{aligned}
$$

\subsubsection{Shaking moment balancing}

Let us now derive the expression of the shaking moment $M^{s h}$, expressed at point $O$, of such a mechanism:

$$
M^{s h}=\sum_{i=1}^{5} m_{i}\left(x_{S_{i}} \ddot{y}_{S_{i}}-y_{S_{i}} \ddot{x}_{S_{i}}+k_{i}^{2} \ddot{\theta}_{i}\right)+\sum_{i=1}^{3} m_{c w_{i}}\left(x_{S_{c w_{i}}} \ddot{y}_{S_{c w_{i}}}-y_{S_{c w_{i}}} \ddot{x}_{S_{c w_{i}}}\right)
$$

where $x_{S_{i}}, y_{S_{i}}, \ddot{x}_{S_{i}}$ and $\ddot{y}_{S_{i}}$ are the position and accelerations along $\mathbf{x}$ and $\mathbf{y}$ axes of points $S_{i}$, respectively, and $x_{S_{c w_{i}}}, y_{S_{c w_{i}}}, \ddot{x}_{S_{c w_{i}}}$ and $\ddot{y}_{S_{c w_{i}}}$ are the position and accelerations along $\mathbf{x}$ and $\mathbf{y}$ axes of points $S_{c w_{i}}$, respectively, $k_{i}$ is the radius of gyration of link $i$.

Now, let us consider as in the previous case, that link 2 is a physical pendulum and that the input speed is constant.

Taking into account (28), one can simplify (51) as:

$$
M^{s h}=I_{3} \ddot{\theta}_{3}+I_{4} \ddot{\theta}_{4}
$$

where

$$
\begin{gathered}
I_{3}=m_{3}\left(k_{3}^{2}+r_{3}^{2}\right)+m_{2} \frac{r_{2}}{l_{2}} l_{3}^{2}+m_{c w_{2}} r_{c w_{2}}^{2}+\left(m_{4}+m_{5}+m_{c w_{3}}\right) l_{3}^{\prime 2} \\
I_{4}=m_{4}\left(k_{4}^{2}+r_{4}^{2}\right)+m_{5} l_{4}^{2}+m_{c w_{3}} r_{c w_{3}}^{2}
\end{gathered}
$$

Introducing (39) into (52), we find:

$$
M^{s h}=\left(I_{3}-I_{4}\right) \ddot{\theta}_{3}
$$

Thus, the mechanism will be moment balanced if:

$$
I_{3}=I_{4}
$$

which can be obtained using a design of link 4 for which the radius of gyration should be equal to

$$
k_{4}=\sqrt{\frac{I_{3}-m_{4} r_{4}^{2}-m_{5} l_{4}^{2}-m_{c w_{3}} r_{c w_{3}}^{2}}{m_{4}}}
$$


It should be mentioned that, in order to avoid the singular configurations of the added structure, the value of angle $\alpha$ should be chosen carefully during the design process.

Let us consider two illustrative examples of the proposed balancing technique.

\subsection{Illustrative examples and numerical simulations}

\subsubsection{Balancing by adding a class-two $R R R$ Assur group}

Let us carry out the complete shaking force and shaking moment balancing of a four-bar linkage with parameters

- $l_{1}=0.2 \mathrm{~m}, l_{2}=0.27 \mathrm{~m}, l_{3}=0.25 \mathrm{~m}, \beta=0 \mathrm{deg}$,

- $r_{1}=0.1 \mathrm{~m}, r_{2}=0.135 \mathrm{~m}, r_{3}=0.125 \mathrm{~m}$,

- $k_{1}=0.056 \mathrm{~m}, k_{2}=0.135 \mathrm{~m}, k_{3}=0.086 \mathrm{~m}$,

- $m_{1}=1 \mathrm{~kg}, m_{2}=1 \mathrm{~kg}, m_{3}=1 \mathrm{~kg}$.

The simulations of the proposed mechanism have been carried out using ADAMS software and the obtained results are shown in Fig. 5 (full line).

Now we add the $R R R$ Assur group with prescribed distribution of the centre of mass and inertia. Its geometric and mass properties are:

- $l_{4}=0.8 \mathrm{~m}, l_{5}=0.25 \mathrm{~m}$,

- $r_{4}=0.4 \mathrm{~m}, r_{5}=0.125 \mathrm{~m}$,

- $m_{4}=1.5 \mathrm{~kg}, m_{5}=1 \mathrm{~kg}$.

and the location and mass of the added counterweights are:

- $r_{c w_{1}}=-0.1 \mathrm{~m}, r_{c w_{2}}=0.25 \mathrm{~m}, r_{c w_{3}}=-0.125 \mathrm{~m}$,

- $m_{c w_{1}}=2 \mathrm{~kg}, m_{c w_{3}}=2.5 \mathrm{~kg}$.

The radii of gyration of elements 4 and 5 and the mass of the counterweight $m_{c w_{2}}$ are not given as they depend on the value of angle $\alpha$ which is not yet fixed (Fig. 3). Their variations as a function of $\alpha$ are shown in Fig. 6. In these figures, the values of $\alpha$ are bounded between 60 and $180 \mathrm{deg}$. in order to avoid the RRR Assur group to cross a singularity during the motion. In Fig. 5 (dotted line), it is shown that after the addition of the Assur group, the shaking force and shaking moment are cancelled.

As angle $\alpha$ is a free parameter that has only influence on the values $k_{4}, k_{5}$ and $m_{c w_{2}}$, it can be chosen so that it minimizes one supplementary criterion. In the remainder of the paper, this criterion is chosen to be the linkage input torque [50,51]. It should however be mentioned that angle $\alpha$ could be used to minimize another criterion such as the power consumption, the energy, etc.

It should also be noted that the input torque $\tau$, i.e. the torque requested by the actuator to move the mechanism, is computed in the two illustrative examples by using the Lagrange equations [51]: 


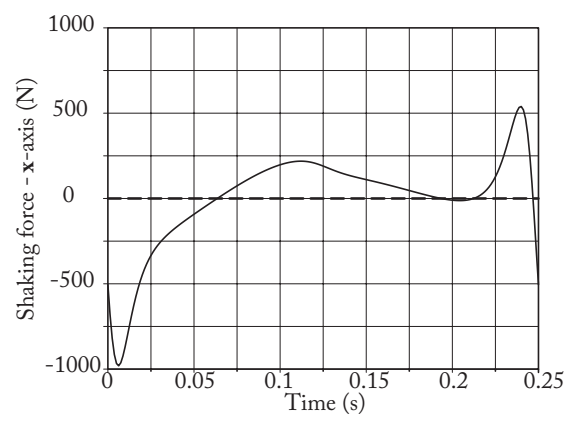

(a) Shaking force along $\mathbf{x}$ axis

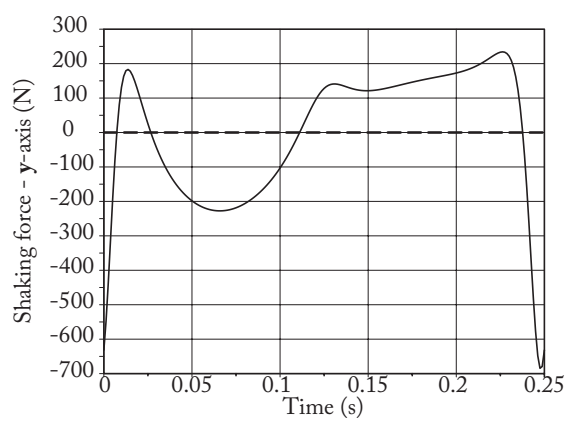

(b) Shaking force along $\mathbf{y}$ axis

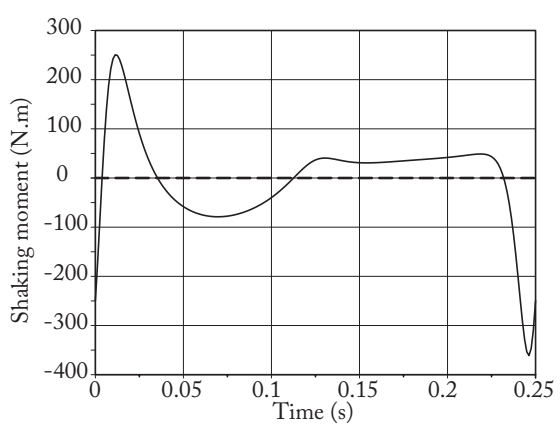

(c) Shaking moment

Fig. 5 Shaking force and shaking moment of the four-bar mechanism before (full line) and after (dotted line) balancing.

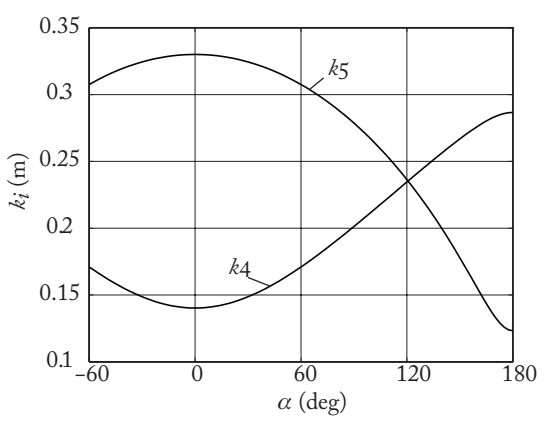

(a) Variation of $k_{4}$ and $k_{5}$

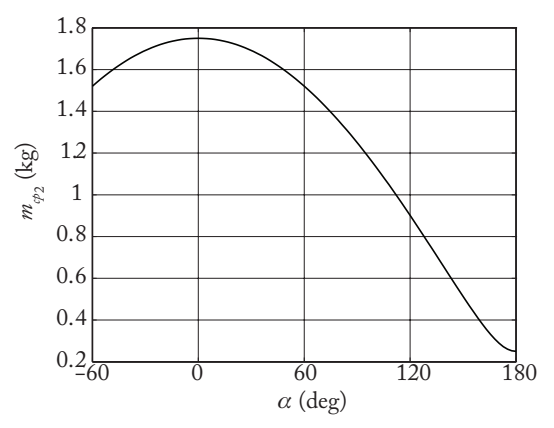

(b) Variation of $m_{c w_{2}}$

Fig. 6 Variation of the values of parameters $k_{4}, k_{5}$ and $m_{c w_{2}}$ as a function of angle $\alpha$.

$$
\tau=\frac{\mathrm{d}}{\mathrm{d} t}\left(\frac{\partial L}{\partial \dot{\theta}_{1}}\right)-\frac{\partial L}{\partial \theta_{1}}
$$




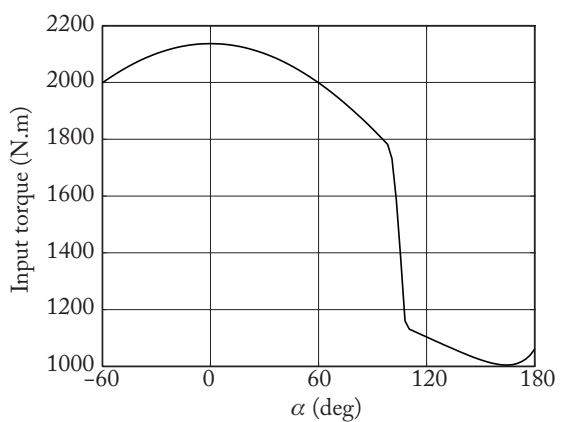

Fig. 7 Variation of the maximal input torque absolute value as a function of angle $\alpha$.

where $L=T-V$ is the Lagrangian of the system, $V$ is the potential energy (equal to 0 in absence of gravity) and $T$ is the kinetic energy:

$$
T=\frac{1}{2} \sum_{i} m_{i}\left(\dot{x}_{S_{i}}^{2}+\dot{y}_{S_{i}}^{2}\right)+\frac{1}{2} \sum_{j} I_{j} \dot{\theta}_{j}^{2}
$$

$\dot{x}_{S_{i}}$ and $\dot{y}_{S_{i}}$ being the velocities along $\mathbf{x}$ and $\mathbf{y}$ axes of any centre of mass (for links and as well as for counterweights).

In Fig. 7, the maximum of the input torque absolute value of as a function of angle $\alpha$ is shown. Thus, it is possible to see that if the value of $\alpha$ is chosen arbitrarily, the input torques can grow up to 2140 N.m (for $\alpha=0$ deg.). It also appears that the input torque will be minimal if $\alpha=164 \mathrm{deg}$. In this case, the value of the input torque is 1010 N.m, i.e. about 2 times less than in the first case.

\subsubsection{Balancing by adding a class-two RRP Assur group}

We now propose obtaining the complete shaking force and shaking moment balancing of the same mechanism by adding a class-two RRP Assur group. Its geometric and mass properties are

- $l_{4}=0.25 \mathrm{~m}, l_{5}=0.25 \mathrm{~m}, \alpha=-90 \mathrm{deg}$,

- $r_{4}=0.125 \mathrm{~m}$,

- $m_{4}=0.35 \mathrm{~kg}, m_{5}=0.1 \mathrm{~kg}$.

and the location and mass of the added counterweights are:

- $r_{c w_{1}}=-0.1 \mathrm{~m}, r_{c w_{2}}=0.25 \mathrm{~m}, r_{c w_{3}}=-0.125 \mathrm{~m}$,

- $m_{c w_{1}}=2 \mathrm{~kg}, m_{c w_{3}}=0.55 \mathrm{~kg}$.

The radii of gyration of element 4 and the mass of the counterweight $m_{c w_{2}}$ are not expressed in these tables as they depend on the value of angle $\alpha$ (Fig. 4). Their variations as a function of $\alpha$ are shown in Fig. 8. In these figures, the values of are bounded between 25 and $100 \mathrm{deg}$. or 205 and $280 \mathrm{deg}$. in order to avoid the 


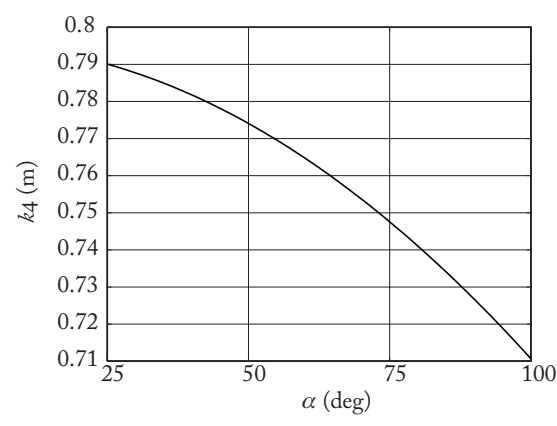

(a) Variation of $k_{4}$ for $\alpha \in\left\{\begin{array}{ll}25 & 100\end{array}\right\} \mathrm{deg}$.

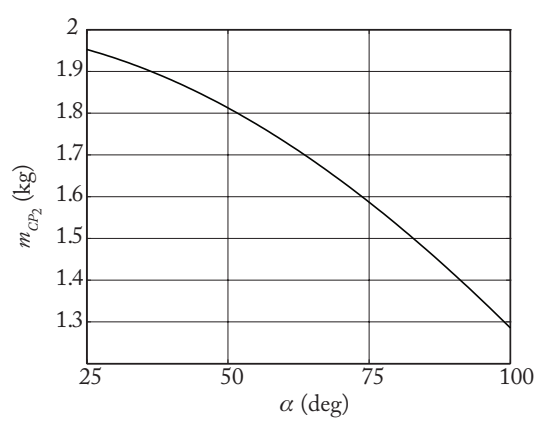

(c) Variation of $m_{c w_{2}}$ for $\alpha \in\left\{\begin{array}{ll}25 & 100\end{array}\right\}$ deg.

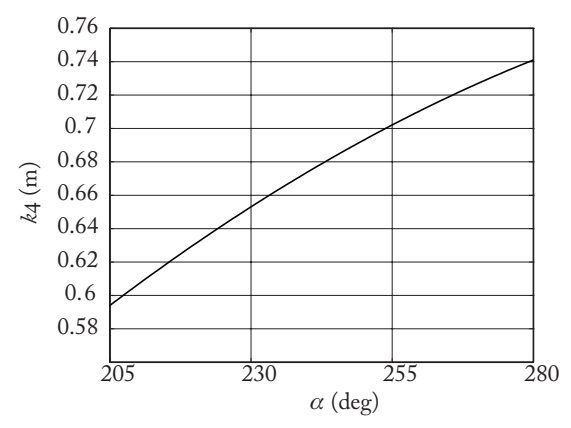

(b) Variation of $k_{4}$ for $\alpha \in\{205280\}$ deg.

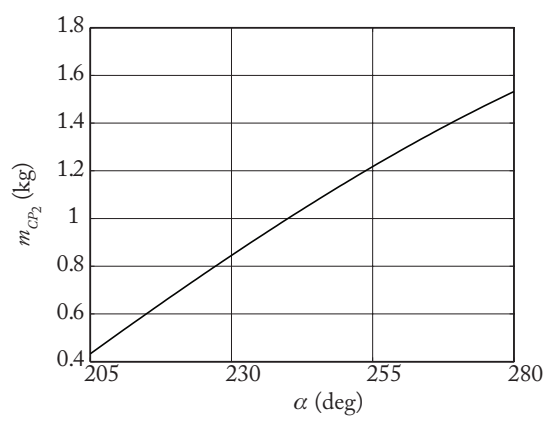

(d) Variation of $m_{c w_{2}}$ for $\alpha \in\{205280\}$ deg.

Fig. 8 Variation of the values of parameters $k_{4}$ and $m_{c w_{2}}$ as a function of angle $\alpha$.

$R R P$ Assur group to cross a singularity during the motion. The simulations of the proposed mechanism have been carried out using ADAMS software and the results are similar to the previous case shown in Fig. 5.

As was mentioned above, the angle $\alpha$ is a not fixed design parameter and it can be found from minimization of the input torque of the mechanism. In Fig. 9, maximum of the input torque absolute value as a function of angle $\alpha$ is shown. It is possible to see that if the value of $\alpha$ is chosen arbitrarily, the input torques can grow up to 2300 N.m (for $\alpha=25 \mathrm{deg}$.). It also appears that the input torque will be minimal if $\alpha=205 \mathrm{deg}$. In this case, the value of the input torque is $1380 \mathrm{~N} . \mathrm{m}$, i.e. about 1.7 times less than in the first case.

Let us now consider the balancing of parallel robots by the addition of one or several class-two RRR Assur groups. 


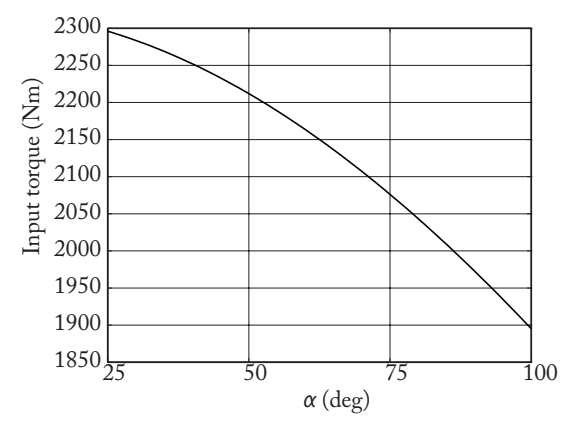

(a) For $\alpha \in\{25100\}$ deg.

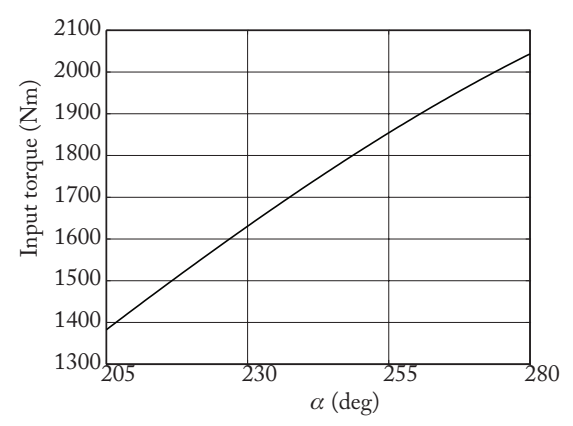

(b) For $\alpha \in\{25100\}$ deg.

Fig. 9 Variation of the maximal input torque absolute value as a function of angle $\alpha$.

\section{Balancing of parallel robots by the addition of one or several class-two RRR Assur groups}

For robots, shaking force balancing is mostly obtained via an optimal redistribution of movable masses [7-9,54-60] or adjustment of kinematic parameters [61]. The cancellation of the shaking moment is a more complicated task and can be obtained using three main different methods:

1. Shaking moment balancing using counter-rotations $[62,63]$,

2. Shaking moment balancing by adding four-bar linkages [45-47, 64, 65] and

3. Shaking moment balancing by optimal trajectory planning [63, 66-69].

Previous works have been devoted to the study of parallel manipulators with revolute joints and few studies has been carried out on complete shaking force and shaking moment balancing of parallel manipulators with prismatic pairs.

In this section, we propose solutions for complete shaking force and shaking moment balancing of planar parallel manipulators with prismatic pairs. We illustrate these solutions via the 3-RPR parallel manipulator. All obtained results are validated using ADAMS software simulations

\subsection{Complete shaking moment and shaking force balancing by adding an idler loop between the base and the platform}

Inertia force balancing by adding an idler loop made of a single $R R R$ Assur group is known to be used for 1 degree of freedom (dof) mechanisms [19,36,70-72]. With regard to planar manipulators, such an approach has only been used in the balancing of gravitational and inertia forces $[59,60,73,74]$. 


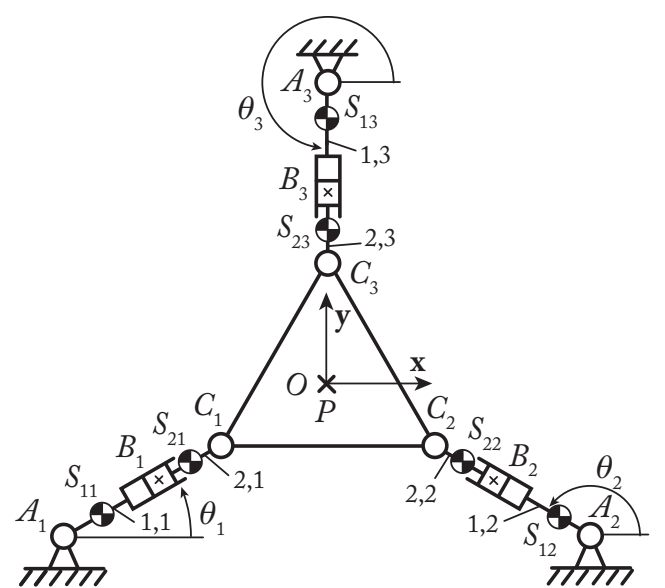

(a) Schematics of the 3-RPR mechanism (for this config- (b) Schematics of the added idler uration, points $O$ and $P$ coincide)

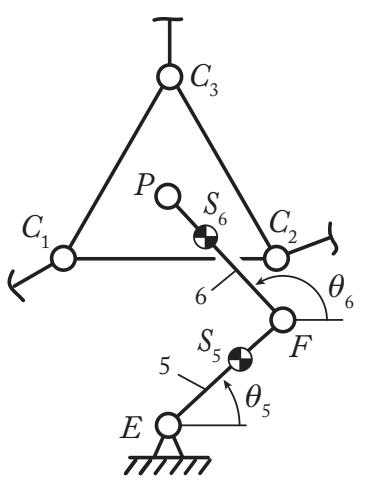

loop (RRR chain)

Fig. 10 Schematic of the $3-R P R$ robot under study.

In this sub-section, the complete shaking force and shaking moment balancing of planar manipulators by adding an idler loop made of a single RRR Assur group is discussed. The added balancing loop is mounted between the base and the platform of the mechanism. We illustrate the suggested balancing technique on a 3-RPR mechanism (Fig. 10). Please note that we do not mention the type of actuation of the mechanism as it has no influence on the balancing.

\subsubsection{Theoretical background}

Firstly, let us analyze the cancellation of the dynamic reactions of the 3-RPR planar parallel mechanism (Fig. 10(a)). Such a mechanism has 3 dof (two translations in the $(O x y)$ plane and one rotation of the moving platform around an axis perpendicular to $(O x y)$ ) and is composed of three identical legs, each being composed of a revolute joint attached to the base at point $A_{i}$ (in the following, $i=1 \cdots 3$ ), one moving prismatic guide, located at point $B_{i}$, and another revolute joint attached to the platform at $C_{i}$. The base and platform triangles, denoted $A_{1} A_{2} A_{3}$ and $C_{1} C_{2} C_{3}$, are equilateral. On this manipulator, typically, the actuated joints are the first revolute joint at $A_{i}$ or the linear guide at $B_{i}$.

Considering that the $\mathbf{x}$ axis is directed along the line $A_{1} A_{2}$, the $\mathbf{y}$ axis being perpendicular to the $\mathbf{x}$ axis and the origin of the base frame located at point $O$, the centre of the circumcircle of triangle $A_{1} A_{2} A_{3}$, one can define the coordinate $x, y$ and $\phi$ of the platform, as being respectively the coordinates of point $P$ along the $\mathbf{x}$ and $\mathbf{y}$ axes and the angle between the line $C_{1} C_{2}$ and $A_{1} A_{2}$. 
Let us denote as $S_{i j}$ the centre of mass of link $i j(j=1,2)$, which has a mass $m_{j}$ and an axial moment of inertia $I_{S_{j}}$. The centre of mass of the platform is located at point $P$. The mass of the platform is $m_{p}$ and its axial moment of inertia $I_{p}$.

In order to cancel the shaking forces and shaking moment of the manipulator, an idler loop is added between the base and the platform (Fig. 10(b)). The centre of mass of elements 5 and 6 of the idler loop are located at $S_{5}$ and $S_{6}$, respectively. Their masses are denoted as $m_{5}$ and $m_{6}$ and their axial moments of inertia as $I_{S_{5}}$ and $I_{S_{6}}$, respectively. The positions of the coms are defined such that $\overrightarrow{A_{i} S_{1 i}}=r_{1} l_{B_{i} C_{i}} \mathbf{u}_{i}$, $\overrightarrow{C_{i} S_{2 i}}=\left(r_{2} 1\right) l_{B_{i} C_{i}} \mathbf{u}_{i}, \overrightarrow{E S_{5}}=r_{5} \overrightarrow{E F}$, and $\overrightarrow{F S_{6 i}}=r_{6} \overrightarrow{F P}, r_{1}, r_{2}, r_{5}$, and $r_{6}$ being dimensionless coefficients, and $\mathbf{u}_{i}$ a unit vector directed along $\overrightarrow{B_{i} C_{i}}$.

The expression of the shaking force $\mathbf{f}^{\text {sh }}$ transmitted by the robot to the ground is:

$$
\mathbf{f}^{s h}=\left(\sum_{i=1}^{3} \sum_{j=1}^{2} m_{j} \ddot{\mathbf{r}}_{S_{i j}}\right)+m_{p} \ddot{\mathbf{r}}_{P}+m_{5} \ddot{\mathbf{r}}_{S_{5}}+m_{6} \ddot{\mathbf{r}}_{S_{6}}
$$

where $\ddot{\mathbf{r}}_{S_{i j}}, \ddot{\mathbf{r}}_{P}, \ddot{\mathbf{r}}_{S_{5}}$ and $\ddot{\mathbf{r}}_{S_{6}}$ are the accelerations of the coms $S_{i j}$, of $P, S_{5}$ and $S_{6}$, respectively.

Developping (60), it can be demonstrated that the shaking force $\mathbf{f}^{\text {sh }}$ can be expressed as:

$$
\begin{aligned}
\mathbf{f}^{s h}= & \left(m_{1} r_{1}-m_{2}\left(1-r_{2}\right)\right) \sum_{i=1}^{3} \mathbf{a}_{i}+\left(3 m_{2}+m_{p}+m_{6} r_{6}\right) \mathbf{a}_{6} \\
& +\left(3 m_{2}+m_{p}+m_{5} r_{5}+m_{6}\right) \ddot{\mathbf{r}}_{F}
\end{aligned}
$$

with

$$
\begin{aligned}
& \mathbf{a}_{i}=l_{B_{i} C_{i}}\left(\ddot{\theta}_{i}\left[\begin{array}{c}
-\sin \theta_{i} \\
\cos \theta_{i}
\end{array}\right]-\dot{\theta}_{i}^{2}\left[\begin{array}{c}
\cos \theta_{i} \\
\sin \theta_{i}
\end{array}\right]\right) \\
& \mathbf{a}_{6}=l_{F P}\left(\ddot{\theta}_{6}\left[\begin{array}{c}
-\sin \theta_{6} \\
\cos \theta_{6}
\end{array}\right]-\dot{\theta}_{6}^{2}\left[\begin{array}{c}
\cos \theta_{6} \\
\sin \theta_{6}
\end{array}\right]\right)
\end{aligned}
$$

At this step, only five counterweights are needed in the cancellation of the shaking force, but it could be demonstrated after more derivations that three others are necessary for the cancellation of the shaking moment. Therefore, we propose directly adding three supplementary counterweights (Fig. 11). The positions of the eight added counterweights are $\overrightarrow{A_{i} M_{1 i}}=r_{c w_{1}} l_{B_{i} C_{i}} \mathbf{u}_{i}, \overrightarrow{B_{i} M_{2 i}}=\left(r_{c w_{2}}-1\right) l_{B_{i} C_{i}} \mathbf{u}_{i}$, $\overrightarrow{E M_{5}}=r_{c w_{5}} \overrightarrow{E F}$, and $\overrightarrow{F M_{6}}=r_{c w_{6}} \overrightarrow{F P}, r_{c w_{1}}, r_{c w_{2}}, r_{c w_{5}}$, and $r_{c w_{6}}$ being dimensionless coefficients. Their masses are respectively denoted $m_{c w_{1}}, m_{c w_{2}}, m_{c w_{5}}$ and $m_{c w_{6}}$. With the addition of the counterweights, the shaking force becomes:

$$
\begin{aligned}
\mathbf{f}^{s h^{*}}= & \mathbf{f}^{s h}+\left(m_{c w_{1}} r_{c w_{1}}-m_{c w_{2}}\left(1-r_{c w_{2}}\right)\right) \sum_{i=1}^{3} \mathbf{a}_{i}+m_{c w_{6}} r_{c w_{6}} \mathbf{a}_{6} \\
& +\left(m_{c w_{5}} r_{c w_{5}}+m_{c w_{6}}\right) \ddot{\mathbf{r}}_{F}
\end{aligned}
$$

Thus, the shaking force is cancelled if: 


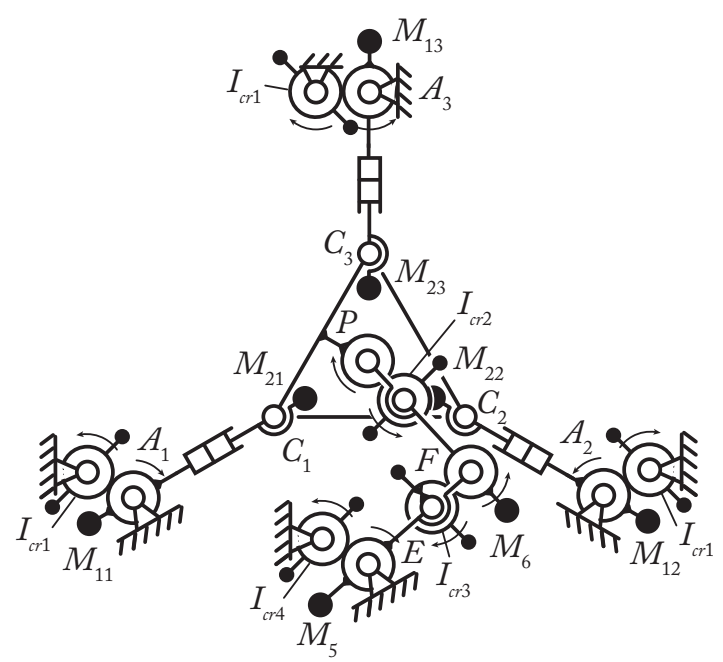

Fig. 11 Schematics of the 3-RPR mechanism with the added RRR chain used for the cancellation of the shaking force and shaking moment.

$$
\begin{aligned}
& m_{c w_{1}}=-\frac{m_{1} r_{1}}{r_{c w_{1}}} \\
& m_{c w_{2}}=-\frac{m_{2}\left(1-r_{2}\right)}{1-r_{c w_{2}}} \\
& m_{c w_{6}}=-\frac{3\left(m_{2}+m_{c w_{2}}\right)+m_{p}+m_{6} r_{6}}{r_{c w_{6}}} \\
& m_{c w_{5}}=-\frac{3\left(m_{2}+m_{c w_{2}}\right)+m_{p}+m_{5} r_{5}+m_{6}+m_{c w_{6}}}{r_{c w_{5}}}
\end{aligned}
$$

The expression of the shaking moment $M_{O}^{s h}$ of the modified structure (expressed at point $O$ ) can be written as:

$$
M_{O}^{s h}=\frac{\mathrm{d}}{\mathrm{d} t} H_{O}
$$

where $H_{O}$ is the angular momentum of the leg (expressed at point $O$ ). Thus, in order to cancel the shaking moment, the angular momentum is held constant over time.

The expression of the angular momentum $H_{O}$ is equal to:

$$
\begin{aligned}
H_{O}= & \sum_{i=1}^{3} \sum_{j=1}^{2}\left(m_{j}\left(x_{S_{i j}} \dot{y}_{S_{i j}}-y_{S_{i j}} \dot{x}_{S_{i j}}\right)+m_{c w_{j}}\left(x_{M_{i j}} \dot{y}_{M_{i j}}-y_{M_{i j}} \dot{x}_{M_{i j}}\right)+I_{S_{j}} \dot{\theta}_{i}\right) \\
& +I_{p} \dot{\phi}+\sum_{j=5}^{6}\left(m_{j}\left(x_{S_{j}} \dot{y}_{S_{j}}-y_{S_{j}} \dot{x}_{S_{j}}\right)+m_{c w_{j}}\left(x_{M_{j}} \dot{y}_{M_{j}}-y_{M_{j}} \dot{x}_{M_{j}}\right)+I_{S_{j}} \dot{\theta}_{j}\right)
\end{aligned}
$$


where $x_{Q}, y_{Q}, \dot{x}_{Q}$ and $\dot{y}_{Q}$ are the position and velocities of any point $Q$ along $\mathbf{x}$ and y axes, respectively ( $Q$ being either point $S_{i j}, M_{i j},(j=1,2), S_{j}$ or $M_{j}(j=5,6)$ ).

Developing and introducing (65) into (67) yields

$$
\begin{aligned}
H_{O}= & \sum_{i=1}^{3}\left(I_{S_{1}}+I_{S_{2}}+\left(m_{1} r_{1}^{2}+m_{c w_{1}} r_{c w_{1}}^{2}+m_{2}\left(1-r_{2}\right)^{2}+m_{c w_{2}}\left(1-r_{c w_{2}}\right)^{2}\right) l_{B_{i} C_{i}}^{2}\right) \dot{\theta}_{i} \\
& +\left(I_{S_{6}}+\left(m_{6} r_{6}^{2}+m_{c w_{6}} r_{c w_{6}}^{2}+m_{p}+3\left(m_{2}+m_{c w_{2}}\right)\right) l_{F P}^{2}\right) \dot{\theta}_{6} \\
& +\left(I_{S_{5}}+\left(m_{5} r_{5}^{2}+m_{c w_{5}} r_{c w_{5}}^{2}+m_{6}+m_{c w_{6}}+m_{p}+3\left(m_{2}+m_{c w_{2}}\right)\right) l_{E F}^{2}\right) \dot{\theta}_{5} \\
& +\left(I_{p}+3\left(m_{2}+m_{c w_{2}}\right) l_{C_{i} P}^{2}\right) \dot{\phi}
\end{aligned}
$$

After such modifications of the $R R R$ chain, the angular momentum of the legs of the mechanism and of the $R R R$ chain can be balanced using six counter-rotations (Fig. 11), which have an axial moment of inertia equal to:

$$
\begin{aligned}
& I_{c r_{1}}=I_{S_{1}}+I_{S_{2}}+\left(m_{1} r_{1}^{2}+m_{c w_{1}} r_{c w_{1}}^{2}+m_{2}\left(1-r_{2}\right)^{2}+m_{c w_{2}}\left(1-r_{c w_{2}}\right)^{2}\right) l_{B_{i} C_{i}}^{2} \\
& I_{c r_{2}}=I_{p}+3\left(m 2+m_{c w_{2}}\right) l_{C_{i} P}^{2} \\
& I_{c r_{3}}=I_{S_{6}}+\left(m_{6} r_{6}^{2}+m_{c w_{6}} r_{c w_{6}}^{2}+m_{p}+3\left(m_{2}+m_{c w_{2}}\right)\right) l_{F P}^{2}+2 I_{c r_{2}} \\
& I_{c r_{4}}=I_{S_{5}}+\left(m_{5} r_{5}^{2}+m_{c w_{5}} r_{c w_{5}}^{2}+m_{6}+m_{c w_{6}}+m_{p}+3\left(m_{2}+m_{c w_{2}}\right)\right) l_{E F}^{2}+2 I_{c r_{3}}
\end{aligned}
$$

\subsubsection{Illustrative examples and numerical simulations}

Let us illustrate the suggested balancing approach using numerical simulations carried out with ADAMS software. For this purpose, non balanced and balanced 3-RPR parallel manipulators will be compared.

The chosen trajectory for simulations is a straight line of the controlled point of the platform, achieved in $t_{f}=0.25 \mathrm{~s}$, between $P_{0}=\left[\begin{array}{ll}x_{0} & y_{0}\end{array}\right]^{T}=\left[\begin{array}{lll}0.05 & \mathrm{~m} & 0 \mathrm{~m}\end{array}\right]^{T}$ and $P_{f}=\left[\begin{array}{ll}x_{f} & y_{f}\end{array}\right]^{T}=\left[\begin{array}{lll}0.2 & m & 0\end{array}\right]^{T}$ with a rotation of the platform from $\phi_{0}=0 \mathrm{deg}$. to $\phi_{f}=30 \mathrm{deg}$. For the displacement of the mechanism, fifth order polynomial laws are used and therefore the trajectory is defined by the following expressions:

$$
\left\{\begin{array}{l}
x(t)=x_{0}+s(t)\left(x_{f}-x_{0}\right) \\
y(t)=0 \\
\phi(t)=\phi_{0}+s(t)\left(\phi_{f}-\phi_{0}\right)
\end{array}\right.
$$

with

$$
s(t)=\frac{10}{t_{f}^{3}} t^{3}-\frac{15}{t_{f}^{4}} t^{4}+\frac{6}{t_{f}^{5}} t^{5}
$$

The parameters used for the simulations are the followings:

- $l_{O E}=0 \mathrm{~m}, l_{O A_{i}}=0.35 \mathrm{~m}, l_{B_{i} C_{i}}=0.05 \mathrm{~m}, l_{C_{i} P}=0.1 \mathrm{~m}, l_{E F}=0.15 \mathrm{~m}$ and $l_{F P}=$ $0.1581 \mathrm{~m}$, 


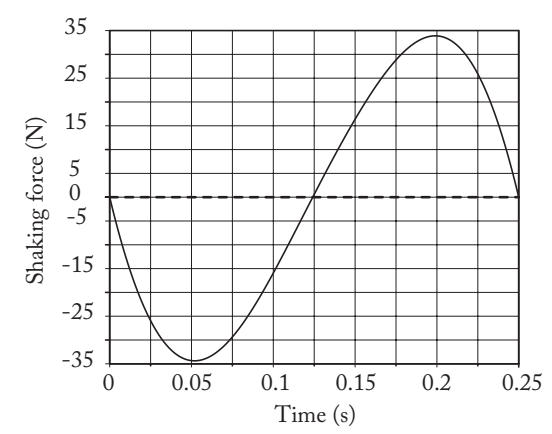

(a) shaking force along $\mathbf{x}$-axis

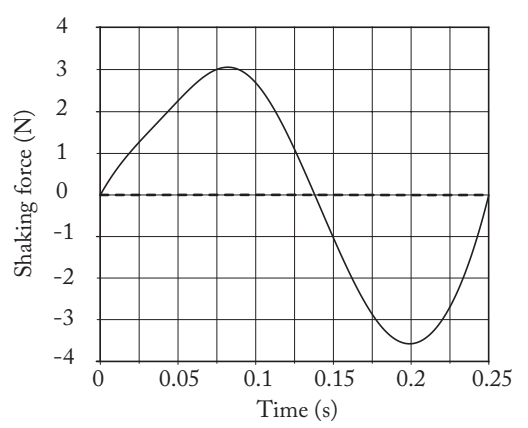

(b) shaking force along $\mathbf{y}$-axis

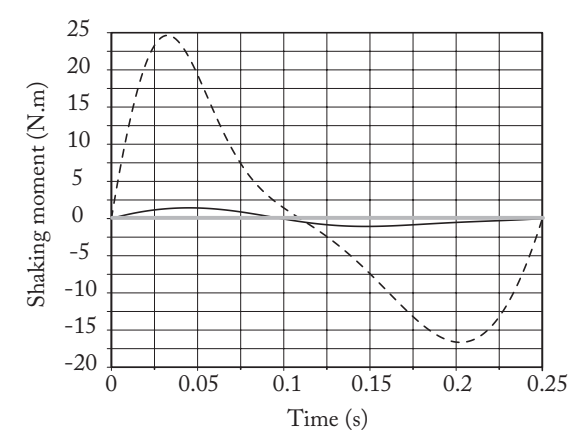

(c) shaking moment around $\mathbf{z}$-axis

Fig. 12 Shaking force and shaking moment before (solid line) and after (dashed line) the addition of the counterweights, and after the addition of the counter-rotations (gray line).

- $r_{1}=2, r_{2}=r_{5}=r_{6}=0.5$,

- $m_{1}=0.75 \mathrm{~kg}, m_{2}=0.37 \mathrm{~kg}, m_{5}=0.42 \mathrm{~kg}, m_{6}=0.47 \mathrm{~kg} ; m_{p}=1 \mathrm{~kg}$,

- $I_{S_{1}}=0.00344 \mathrm{~kg} \cdot \mathrm{m}^{2}, I_{S_{2}}=0.00025 \mathrm{~kg} \cdot \mathrm{m}^{2}, I_{S_{5}}=0.00122 \mathrm{~kg} \cdot \mathrm{m}^{2}$, $I_{S_{6}}=0.00146 \mathrm{~kg} \cdot \mathrm{m}^{2}, I_{p}=0.00436 \mathrm{~kg} \cdot \mathrm{m}^{2}$.

For such parameters and such a trajectory, the shaking force and shaking moment are computed using ADAMS software and are presented in Fig. 12 (solid line). Then, we add the counterweights and the idler loop EFP to the mechanism. The position coefficients of the counterweights are all equal to $r_{c w_{j}}=-0.5(j=1,2,5,6)$. Therefore, the added masses are equal to $m_{c w_{1}}=0.75 \mathrm{~kg}, m_{c w_{2}}=0.37 \mathrm{~kg}, m_{c w_{5}}=$ $6.92 \mathrm{~kg}, m_{c w_{6}}=21.66 \mathrm{~kg}$. The new values of the shaking force and moment are presented in Fig. 12 (dashed line). It is possible to see that with the added counterweights the shaking efforts are cancelled, while the maximal value of shaking moment is increased by a factor 17 . Finally, we add the counter-rotations. Their values are equal to $I_{c r_{1}}=0.01917 \mathrm{~kg} . \mathrm{m}^{2}, I_{c r_{2}}=0.02665 \mathrm{~kg} \cdot \mathrm{m}^{2}, I_{c r_{3}}=0.18169 \mathrm{~kg} \cdot \mathrm{m}^{2}$, $I_{c r_{4}}=0.72781 \mathrm{~kg} \cdot \mathrm{m}^{2}$. With such counter-rotations, the shaking moment is balanced (in gray line in Fig. 12(c)). 


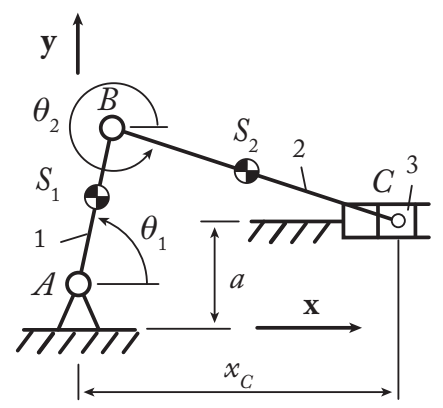

Fig. 13 A general slider-crank mechanism.

\subsection{Complete shaking force and shaking moment balancing using Scott-Russell mechanism}

In this sub-section, another approach for complete shaking force and shaking moment balancing is developed, which consists of adding Scott-Russell mechanisms (mechanisms made of $R R R$ Assur groups) to each leg of the initial architecture of a manipulator. This approach enables a reduction in the number of counter-rotations.

\subsubsection{Properties of the Scott-Russell mechanism}

Let us observe a simple slider-crank mechanism (Fig. 13). The centre of mass of link $i(i=1,2,3)$ is denoted as $S_{i}$. Link $i$ has a mass $m_{i}$ and an axial moment of inertia $I_{S_{i}}$. The positions of the centres of mass are $\overrightarrow{A S_{1}}=r 1 \overrightarrow{A B}, \overrightarrow{B S_{2}}=r_{2} \overrightarrow{B C}, \overrightarrow{C S_{3}}=l_{3} r_{3} \mathbf{x}$, $r_{1}, r_{2}$ and $r_{3}$ being dimensionless coefficients, and $l_{3}$ being a constant length.

It is known that the compete shaking force and shaking moment balancing of a general slider-crank mechanism can be obtained by adding two counterweights mounted on the links and two pairs of counter-rotations. However, it is possible to balance this mechanism without counter-rotation if it has specific geometrical parameters, as in Scott-Russell mechanisms ( $a=0 \mathrm{~m}, l_{A B}=l_{B C}-$ Fig. 13).

Let us consider the balancing of this mechanism. The expression of the shaking force $\mathbf{f}^{s h}$ of a slider-crank mechanism can be written as:

$$
\mathbf{f}^{s h}=\sum_{i=1}^{3} m_{i} \ddot{\mathbf{r}}_{S_{i}}=\left(m_{1} r_{1}+m_{2}\right) \ddot{\mathbf{r}}_{B}+\left(m_{2} r_{2}+m_{3}\right) \mathbf{a}
$$

with

$$
\mathbf{a}=l_{B C}\left(\ddot{\theta}_{2}\left[\begin{array}{c}
-\sin \theta_{2} \\
\cos \theta_{2}
\end{array}\right]-\dot{\theta}_{2}^{2}\left[\begin{array}{c}
\cos \theta_{2} \\
\sin \theta_{2}
\end{array}\right]\right)
$$

$\ddot{\mathbf{r}}_{B}$ being the acceleration of point $B$. 
The constant terms of (72) can be cancelled by the addition of two counterweights located at $M_{j},(j=1,2)$ (Fig. 13), whose masses are denoted as $m_{c w_{j}}$. Their positions are equal to: $\overrightarrow{A M_{1}}=r_{c w_{1}} \overrightarrow{A B}, \overrightarrow{B M_{2}}=r_{c w_{1}} \overrightarrow{B C}, r_{c w_{1}}$ and $r_{c w_{2}}$ being dimensionless coefficients. With the addition of the counterweights, the shaking force becomes:

$$
\mathbf{f}^{s h^{*}}=\mathbf{f}^{s h}+\left(m_{c w_{1}} r_{c w_{1}}+m_{c w_{2}}\right) \ddot{\mathbf{r}}_{B}+m_{c w_{2}} r_{c w_{2}} \mathbf{a}
$$

Thus, the shaking force is balanced if:

$$
m_{c w_{2}}=-\frac{m_{2} r_{2}+m_{3}}{r_{c w_{2}}} \text { and } m_{c w_{1}}=-\frac{m_{1} r_{1}+m_{2}+m_{c w_{2}}+m_{3}}{r_{c w_{1}}}
$$

The expression of the angular momentum $H_{A}$ (expressed at point $A$ ) is:

$$
H_{A}=\sum_{j=1}^{3}\left(m_{j}\left(x_{S_{j}} \dot{y}_{S_{j}}-y_{S_{j}} \dot{x}_{S_{j}}\right)\right)+\sum_{j=1}^{2}\left(m_{c w_{j}}\left(x_{M_{j}} \dot{y}_{M_{j}}-y_{M_{j}} \dot{x}_{M_{j}}\right)+I_{S_{j}} \dot{\theta}_{j}\right)
$$

where $x_{Q}, y_{Q}, \dot{x}_{Q}$ and $\dot{y}_{Q}$ are the position and velocities of any point $Q$ along $\mathbf{x}$ and y axes, respectively ( $Q$ being either point $S_{j}$ or $M_{j},(j=1,2,3)$ ).

Developing and introducing (75) into (76),

$$
\begin{aligned}
H_{A}= & \left(I_{S_{1}}+\left(m_{1} r_{1}^{2}+m_{c w_{1}} r_{c w_{1}}^{2}+m_{2}+m_{c w_{2}}+m_{3}\right) l_{A B}^{2}\right) \dot{\theta}_{1} \\
& +\left(I_{S_{2}}+\left(m_{2} r_{2}^{2}+m_{c w_{1}} r_{c w_{1}}^{2}+m_{3}\right) l_{B C}^{2}\right) \dot{\theta}_{2}
\end{aligned}
$$

with

$$
\dot{\theta}_{2}=-\frac{\dot{y}_{B}\left(x_{C}-x_{B}\right)+\left(a-y_{B}\right)\left(\dot{x}_{C}-\dot{x}_{B}\right)}{l_{B C}^{2}}
$$

where $x_{B}, y_{B}, x_{C}$ are the coordinates along $\mathbf{x}$ and $\mathbf{y}$ axes of points $B$ and $C$, respectively, and $\dot{x}_{B}, \dot{y}_{B}, \dot{x}_{C}$ their velocities.

In order to cancel the shaking moment $M_{A}^{s h}$, the angular momentum has to be constant or null. Developing (77), $M_{A}^{s h}$ can be cancelled if $a=0$ and $l_{A B}=l_{B C}$ (in such a case, $\dot{\theta}_{1}=-\dot{\theta}_{2}$ ) and if:

$$
\begin{aligned}
& I_{S_{1}}+\left(m_{1} r_{1}^{2}+m_{c w_{1}} r_{c w_{1}}^{2}+m_{2}+m_{c w_{2}}+m_{3}\right) l_{A B}^{2} \\
& -I_{S_{2}}-\left(m_{2} r_{2}^{2}+m_{c w_{1}} r_{c w_{1}}^{2}+m_{3}\right) l_{B C}^{2}=0
\end{aligned}
$$

\subsubsection{Balancing of a manipulator's leg using a Scott-Russell mechanism}

Now let us consider a manipulator's leg with an added Scott-Russell mechanism (i.e. an additional $R R R$ Assur group - Fig. 14). Let us denote as $S_{4}$ the centre of mass of link 4, which has a mass $m_{4}$ and an axial moment of inertia $I_{S_{4}}$. The position of $S_{4}$ is such that: $\overrightarrow{A S_{4}}=l_{3} r_{4} \mathbf{u}, r_{4}$ being a dimensionless coefficient and $\mathbf{u}$ a unit vector along $\overrightarrow{\mathrm{CS}_{3}}$.

Now the shaking force becomes: 


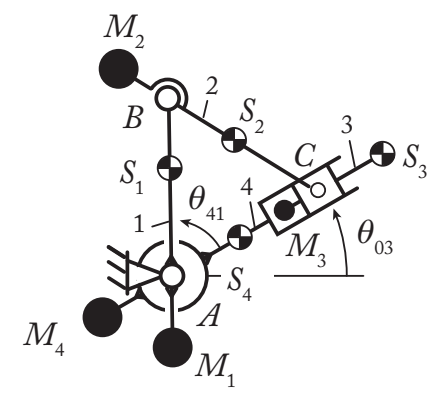

Fig. 14 A manipulator leg with added Scott-Russell mechanism.

$$
\begin{aligned}
\mathbf{f}^{s h}= & \left(m_{1} r_{1}+m_{c w_{1}} r_{c w_{1}}+m_{2}+m_{c w_{2}}+m_{3}\right) \ddot{\mathbf{r}}_{B}+\left(m_{3} r_{3}+m_{4} r_{4}\right) \mathbf{a}_{1} \\
& +\left(m_{2} r_{2}+m_{c w_{2}} r_{c w_{2}}+m_{3}\right) \mathbf{a}_{2}
\end{aligned}
$$

with

$$
\begin{aligned}
& \mathbf{a}_{1}=l_{3}\left(\ddot{\theta}_{03}\left[\begin{array}{c}
-\sin \theta_{03} \\
\cos \theta_{03}
\end{array}\right]-\dot{\theta}_{03}^{2}\left[\begin{array}{c}
\cos \theta_{03} \\
\sin \theta_{03}
\end{array}\right]\right) \\
& \mathbf{a}_{2}=l_{A B}\left(\left(\ddot{\theta}_{03}-\ddot{\theta}_{41}\right)\left[\begin{array}{c}
-\sin \left(\theta_{03}-\theta_{41}\right) \\
\cos \left(\theta_{03}-\theta_{41}\right)
\end{array}\right]-\left(\dot{\theta}_{03}-\dot{\theta}_{41}\right)^{2}\left[\begin{array}{c}
\cos \left(\theta_{03}-\theta_{41}\right) \\
\sin \left(\theta_{03}-\theta_{41}\right)
\end{array}\right]\right)
\end{aligned}
$$

At this step, only one supplementary counterweight is necessary for the cancellation of the shaking force, but it could be demonstrated after more derivations that another is necessary for the cancellation of the shaking moment. Therefore, we propose adding this additionnal counterweight directly. The two counterweights are located at points $M_{3}$ and $M_{4}$, defined such that: $\overrightarrow{C M_{3}}=r_{c w_{3}} l_{3} \mathbf{u}, \overrightarrow{A M_{4}}=r_{c w_{4}} l_{3} \mathbf{u}, r_{c w_{3}}$ and $r_{c w_{4}}$ being dimensionless coefficients. Their masses are respectively denoted $m_{c w_{3}}$ and $m_{c w_{4}}$. With the addition of the counterweights, the shaking force becomes:

$$
\mathbf{f}^{s h^{*}}=\mathbf{f}^{\mathbf{s} h}+m_{c w_{3}} \ddot{\mathbf{r}}_{B}+m_{c w_{3}} \mathbf{a}_{1}+\left(m_{c w_{3}} r_{c w_{3}}+m_{c w_{4}} r_{c w_{4}}\right) \mathbf{a}_{2}
$$

Thus, the shaking force is cancelled if:

$$
\begin{aligned}
m_{c w_{4}} & =-\frac{m_{4} r_{4}}{r_{c w_{4}}} \\
m_{c w_{3}} & =-\frac{m_{3} r_{3}}{r_{c w_{3}}} \\
m_{c w_{2}} & =-\frac{m_{2} r_{2}+m_{3}+m_{c w_{3}}}{r_{c w_{2}}} \\
m_{c w_{1}} & =-\frac{m_{1} r_{1}+m_{2}+m_{c w_{2}}+m_{3}+m_{c w_{3}}}{r_{c w_{1}}}
\end{aligned}
$$




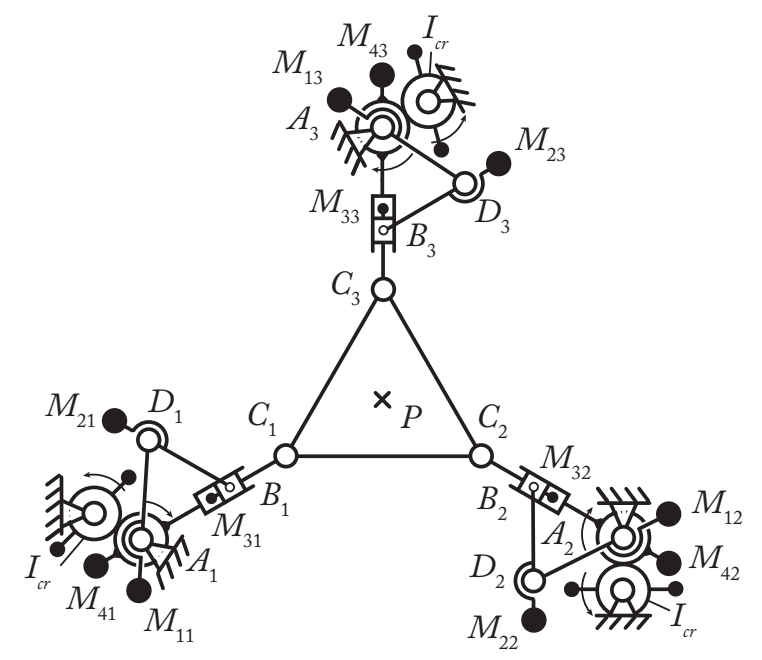

Fig. 15 Schematics of a shaking force and shaking moment balanced 3-RPR mechanism.

Developing and simplifying, the expression of the angular momentum is equal to:

$$
H_{A}=I_{e q_{1}} \dot{\theta}_{03}+I_{e q_{2}} \dot{\theta}_{41}
$$

with

$$
\begin{gathered}
I_{e q_{1}}=\sum_{i=1}^{4} I_{S_{i}}+\left(m_{1} r_{1}^{2}+m_{c w_{1}} r_{c w_{1}}^{2}+m_{2}\left(1-r_{2}\right)^{2}+m_{c w_{2}}\left(1-r_{c w_{2}}\right)^{2}\right) l_{A B}^{2} \\
+\left(m_{3} r_{3}^{2}+m_{c w_{3}} r_{c w_{3}}^{2}+m_{4} r_{4}^{2}+m_{c w_{4}} r_{c w_{4}}^{2}\right) l_{3}^{2} \\
I_{e q_{2}}=I_{S_{1}}+\left(m_{1} r_{1}^{2}+m_{c w_{1}} r_{c w_{1}}^{2}+m_{2}+m_{c w_{2}}+m_{3}\right) l_{A B}^{2} \\
-I_{S_{2}}-\left(m_{2} r_{2}^{2}+m_{c w_{2}} r_{c w_{2}}^{2}+m_{3}\right) l_{B C}^{2}
\end{gathered}
$$

From (79), $I_{e q_{2}}=0$. Therefore, the shaking moment of the leg can be cancelled using a simple counter-rotation $I_{c r}$ with an axial moment of inertia equal to $I_{e q_{1}}$.

\subsubsection{Shaking moment and shaking force balancing of the 3-RPR manipulator}

Now, let us apply such an approach to the 3-RPR mechanism. First of all, let us substitute the platform mass by three points masses located at $C_{1}, C_{2}$ and $C_{3}$, with the values of mass equal to $m_{p_{1}}, m_{p_{2}}$ and $m_{p_{3}}$ respectively $[38,73,75]$. Such a condition can be obtained if:

$$
m_{p_{i}}=m_{p} / 3 \text { and } I_{p}=3 m_{p_{i}} l_{C_{i} P}^{2}
$$


Such a decomposition of the platform enables us to consider the shaking force and shaking moment balancing of each leg of the mechanism. Then, modifying each leg in order to obtain a mechanism similar to a Scott-Russel linkage, the shaking force and shaking moment are cancelled if:

$$
\begin{aligned}
m_{c w_{4}} & =-\frac{m_{4} r_{4}}{r_{c w_{4}}} \\
m_{c w_{3}} & =-\frac{m_{3} r_{3}+m_{p_{i}}}{r_{c w_{3}}} \\
m_{c w_{2}} & =-\frac{m_{2} r_{2}+m_{3}+m_{c w_{3}}+m_{p_{i}}}{r_{c w_{2}}} \\
m_{c w_{1}} & =-\frac{m_{1} r_{1}+m_{2}+m_{c w_{2}}+m_{3}+m_{c w_{3}}+m_{p_{i}}}{r_{c w_{1}}} \\
0 & =I_{S_{1}}+\left(m_{1} r_{1}^{2}+m_{c w_{1}} r_{c w_{1}}^{2}+m_{2}+m_{c w_{2}}+m_{3}\right) l_{A_{i} B_{i}}^{2} \\
& -I_{S_{2}}-\left(m_{2} r_{2}^{2}+m_{c w_{2}} r_{c w_{2}}^{2}+m_{3}\right) l_{B_{i} C_{i}}^{2}, \text { and } \\
I_{c r}= & \sum_{i=1}^{4} I_{S_{i}}+\left(m_{1} r_{1}^{2}+m_{c w_{1}} r_{c w_{1}}^{2}+m_{2}\left(1-r_{2}\right)^{2}+m_{c w_{2}}\left(1-r_{c w_{2}}\right)^{2}\right) l_{A_{i} B_{i}}^{2} \\
& +\left(m_{3} r_{3}^{2}+m_{c w_{3}} r_{c w_{3}}^{2}+m_{4} r_{4}^{2}+m_{c w_{4}} r_{c w_{4}}^{2}+m_{p_{i}}\right) l_{3}^{2}
\end{aligned}
$$

taking into account that $I_{c r}$ is the axial moment of inertia of the counter-rotations (Fig. 15).

Thus, with this approach it is possible to create a fully-balanced shaking force and shaking moment 3-RPR mechanism with only three counter-rotations (Fig. 15), i.e., this method enables a reduction in the number of counter-rotations by a factor of two.

\subsubsection{Illustrative examples and numerical simulations}

The parameters used for the simulations are the followings:

- $l_{O A_{i}}=0.35 \mathrm{~m}, l_{A_{i} B_{i}}=l_{B_{i} C_{i}}=0.25 \mathrm{~m}, l_{C_{i} P}=0.1 \mathrm{~m}$ and $l_{3}=0.025 \mathrm{~m}$,

- $r_{1}=r_{2}=0.5, r_{3}=0$ and $r_{4}=4$,

- $m_{1}=1.09 \mathrm{~kg}, m_{2}=1.1 \mathrm{~kg}, m_{3}=0.37 \mathrm{~kg}, m_{4}=0.75 \mathrm{~kg} ; m_{p}=1 \mathrm{~kg}$,

- $I_{S_{1}}=0.00738 \mathrm{~kg} \cdot \mathrm{m}^{2}, I_{S_{2}}=0.58389 \mathrm{~kg} \cdot \mathrm{m}^{2}, I_{3}=0.00344 \mathrm{~kg} \cdot \mathrm{m}^{2}$, $I_{S_{4}}=0.00025 \mathrm{~kg} \cdot \mathrm{m}^{2}$, and $I_{p}=0.01 \mathrm{~kg} \cdot \mathrm{m}^{2}$.

For these new parameters and for the trajectory used in Section 3.1, taking into account that the position coefficients of the counterweights are equal to $r_{c w_{j}}=-0.5$ $(j=1,3,4), r_{c w_{2}}=-1$, the new values of the counterweights are: $m_{c w_{1}}=3.17 \mathrm{~kg}$, $m_{c w_{2}}=11.71 \mathrm{~kg}, m_{c w_{3}}=0.33 \mathrm{~kg}, m_{c w_{4}}=0.75 \mathrm{~kg}$. The shaking force and shaking moment are then computed (dashed line in Fig. 16). Finally, we add the counter-rotations. Their values are equal to $I_{c r}=1.56907 \mathrm{~kg} . \mathrm{m}^{2}$. With such counterrotations, the shaking moment is balanced (gray line in Fig. 16(c)). 


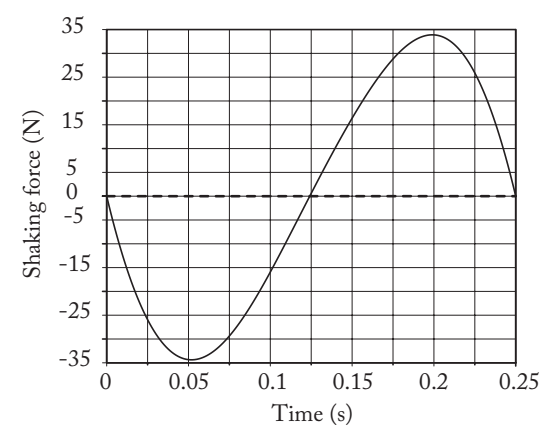

(a) shaking force along $\mathbf{x}$-axis

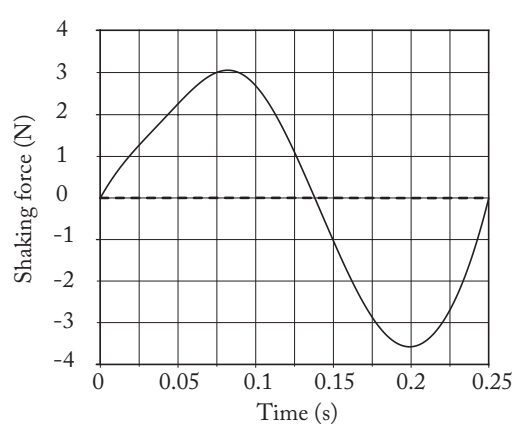

(b) shaking force along $\mathbf{y}$-axis

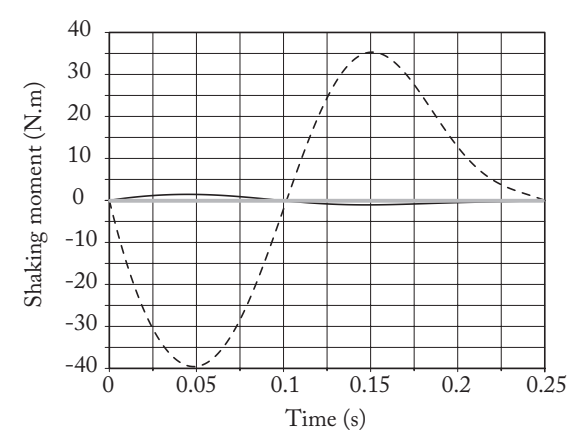

(c) shaking moment around $\mathbf{z}$-axis

Fig. 16 Shaking force and shaking moment before (solid line) and after (dashed line) the addition of the counterweights, and after the addition of the counter-rotations (gray line).

Finally, it should be noted that the combination of the proposed two techniques of balancing enables the creation of fully balanced parallel manipulators with modified legs. As examples, different structures of balanced manipulators are presented in Fig. 17 (3-RPR, 3-PRR and 3-PRP) in which one leg with a prismatic pair is replaced by a leg with only revolute joints. Such a modification allows the displacement of the centre of mass of the manipulator to $C_{3}$ and then to balance the manipulator via the modified leg $C_{3} B_{3} A_{3}$.

In the same way, it is possible to balance a parallel manipulator with prismatic pairs by adding fewer Scott-Russell mechanisms. The balancing schemes for several parallel manipulators are presented in Fig. 18.

Thus, it has been presented new balancing schemes for the shaking force and shaking moment of planar parallel manipulators whose legs are made of prismatic pairs.

Usually, the balancing of parallel manipulators with prismatic pairs is only attained via a considerably complicated design. This section showed that it is possible to balance planar parallel mechanisms using Scott-Russell mechanisms. Such an approach enables a division of the number of counter-rotations by two. Numerical 


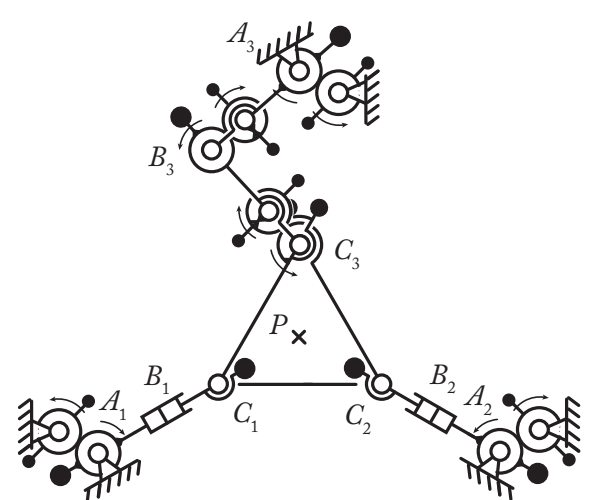

(a) Balanced 3-RPR parallel manipulator

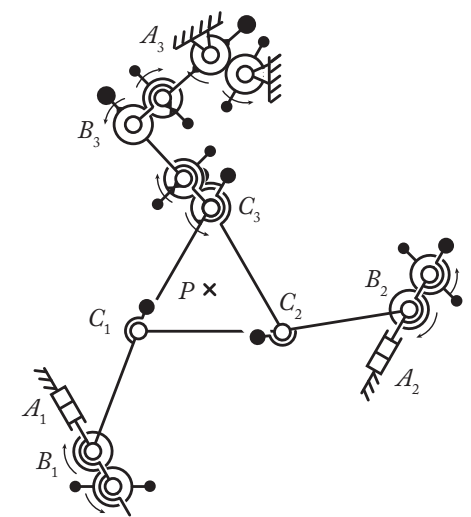

(b) Balanced 3-PRR parallel manipulator

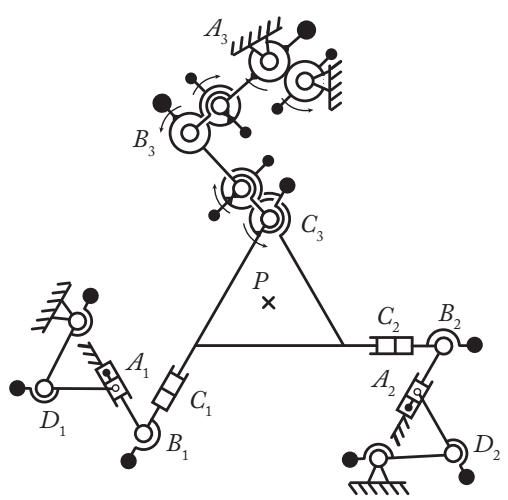

(c) Balanced 3-PRP parallel manipulator

Fig. 17 Complete shaking force and shaking moment balancing of planar manipulators with prismatic pairs via structural modification of one leg.

simulations carried out using ADAMS software validated the obtained results and illustrated that the suggested balancing enables the creation of a parallel manipulator transmitting no inertia load to its base.

\section{Conclusions}

For all balancing method, the main challenge is the trade-off between the complexity of the balanced mechanism and the quality of balancing. In the present Chapter, we have proposed a solution which allows the reduction of the balancing complexity by comparison with the usual approaches. The idea was to slightly modify the mechanism design by adding to it Assur groups, i.e. groups which dos not add any 


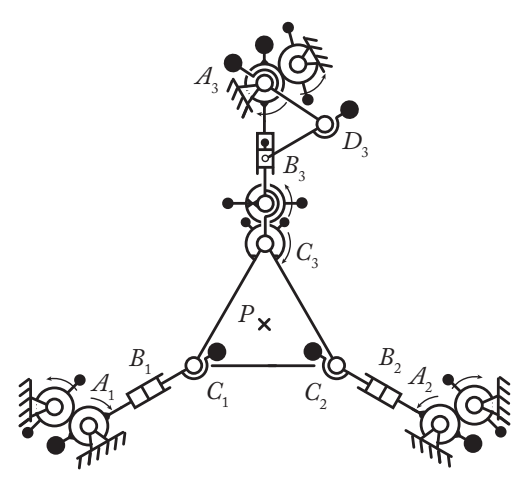

(a) Balancing of 3-RPR parallel manipulator

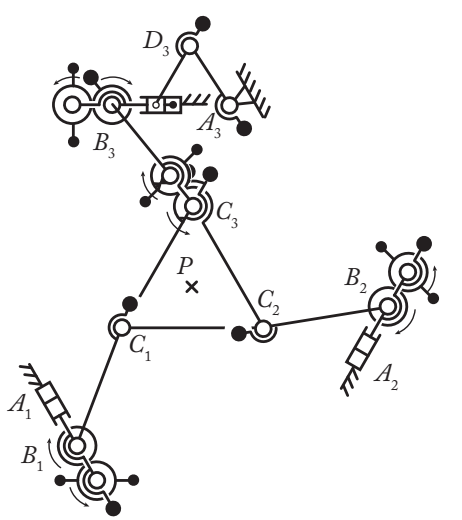

(b) Balancing of 3-PRR parallel manipulator

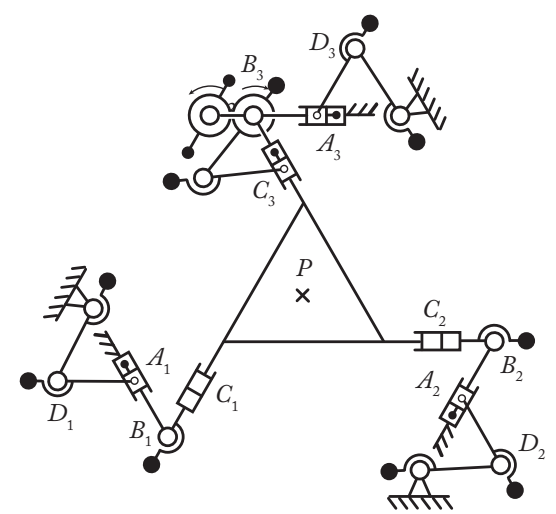

(c) Balancing of 3-PRP parallel manipulator

Fig. 18 Complete shaking force and shaking moment balancing of planar manipulators with prismatic with reduced number of Scott-Russell mechanisms.

supplementary degree of freedom into the mechanism. The use of such a solution was detailed for the shaking force and shaking moment balancing of:

- the in-line four-bar linkage

- the planar parallel robots with prismatic pairs.

For both types of mechanisms, the proposed solution allowed the reduction (or even the cancellation in the case of the four-bar linkage) of the number of counterrotations used for obtaining the shaking moment balancing, which decreases the design complexity and the inherent problems due to the use of counter-rotations (backlash, noise, vibrations, etc.).

All theoretical developments were validated via simulations carried out using ADAMS software. The simulations showed that the obtained mechanisms transmit- 
ted no inertia loads to their surroundings, i.e. the sum of all ground bearing forces and their moments were eliminated.

\section{References}

1. S. Foucault and C.M. Gosselin. On the development of a planar 3-dof reactionless parallel mechanism. In Proceedings of the ASME Design Engineering Technical Conferences and Computers and Information in Engineering Conference (IDECT/CIE 2002), Montreal, Canada, sep 2002.

2. V. van der Wijk, B. Demeulenaere, C.M. Gosselin, and J.L. Herder. Comparative analysis for low-mass and low-inertia dynamic balancing of mechanisms. ASME Journal of Mechanisms and Robotics, 4, 2012.

3. R. Raaijmakers. Besi zoekt snelheidslimiet pakken en plaatsen op (besi attacks the speedlimit for pick and place motion). Mechatronica nieuws (Dutch Magazine), pages 26-31, 2007.

4. G.G. Lowen and R.S. Berkof. Survey of investigations into the balancing of linkages. ASME Journal of Mechanisms, 3:221-231, 1968.

5. K. Ishida and T. Matsuda. Performance characteristics and working comfortableness of forest workers of a new non-vibrating chain saw utilizing perfectly balanced rotation-reciprocation device. In Proceedings of the fifth World Congress of Theory of Machines and Mechanisms, pages 951-954, Montreal, Canada, 1979.

6. V. Arakelian and S. Briot. Balancing of Linkages and Robot Manipulators - Advanced Methods with Illustrative Examples. Springer, 2014.

7. G.G. Lowen, F.R. Tepper, and R.S. Berkof. Balancing of linkages - an update. Mechanism and Machine Theory, 18(3):213-230, 1983.

8. V. Arakelian, M. Dahan, and M.R. Smith. A historical review of the evolution of the theory on balancing of mechanisms. In M. Ceccarelli, editor, Proceedings of the International Symposium on History of Machines and Mechanisms (HMM 2000), pages 291-300. Kluwer Academic Publishers, 2000.

9. V. Arakelian and M.R. Smith. Shaking force and shaking moment balancing of mechanisms: a historical review with new examples. ASME Journal of Mechanical Design, 127:334-339, 2005.

10. V. Nabat, F. Pierrot, M.R. Mijangos, J.M.A. Arteche, R.B. Zabalo, O. Company, and K.F. Perez De Armentia. High-speed parallel robot with four degrees of freedom, patent, 2006.

11. V. van der Wijk, S. Krut, F. Pierrot, and J.L. Herder. Design and experimental evaluation of a dynamically balanced redundant planar 4-RRR parallel manipulator. The International Journal of Robotics Research, 32:744-759, 2013.

12. T. Leinonen. Terminology for the theory of machines and mechanisms. Mechanism and Machine Theory, 26, 1991.

13. O. Fischer. über die reduzierten Systeme und die Hauptpunkte der Glieder eines Gelenkmechanismus. Zeit. für Math. and Phys., 47:429-466, 1902.

14. I.I. Artobolevsky and B.V. Edelshtein. Methods of inertia calculation for mechanisms of agricultural machines. Selkhozizdate, Moscow, 1935. in Russian.

15. F.R.E. Crossley. Dynamics in Machines. Roland Press, New York, 1954.

16. G.L. Talbourdet and P.R. Shepler. Mathematical solution of 4-bar linkages - IV. Balancing of linkages. Machine Design, 13:73-77, 1941.

17. M.R. Smith and L. Maunder. Inertia forces in a four-bar linkage. Mechanical Engineering Science, 9(3):218-225, 1967.

18. R.S. Berkof and G.G. Lowen. A new method for completely force balancing simple linkages. ASME Journal of Engineering for Industry, 91(1):21-26, 1969.

19. H. Hilpert. Weight balancing of precision mechanical instruments. Mechanisms, 3(4):289302, 1968. 
20. G.G. Lowen and R.S. Berkof. Determination of force-balanced four-bar linkages with optimum shaking moment characteristics. ASME Journal of Engineering for Industry, 93(1):3946, 1971.

21. G.G. Lowen and R.S. Berkof. Theory of shaking moment optimization of force-balanced four-bar linkages. ASME Journal of Mechanical Design, 12, 1970.

22. R.S Berkof and G.G. Lowen. Theory of shaking moment optimization of force-balanced fourbar linkages. ASME Journal of Engineering for Industry, 93(1):53-60, 1971.

23. J.L. Wiederrich and B. Roth. Momentum balancing of four-bar linkages. ASME Journal of Mechanical Design, 98B(4):1289-1295, 1976.

24. J.L. Elliot and D. Tesar. The theory of torque, shaking force and shaking moment balancing of four link mechanisms. ASME Journal of Engineering for Industry, 99(3):715-722, 1977.

25. W.L. Carson and J.M. Stephens. Feasible parameter design spaces for force and root-meansquare moment balancing on in-line 4R 4-bar linkage synthesized for kinematic criteria. Mechanism and Machine Theory, 13(6):649-658, 1978.

26. R.S. Haines. Minimum RMS shaking moment or driving torque of a force-balanced 4-bar linkage using feasible counterweights. Mechanism and Machine Theory, 16:185-190, 1981.

27. V.A. Shchepetilnikov. The determination of the mass centers of mechanisms in connection with the problem of mechanism balancing. Mechanisms, 3:367-389, 1968.

28. V. Arakelian and M. Dahan. Partial shaking moment balancing of fully force balanced linkages. Mechanism and Machine Theory, 36(11-12):1241-1252, 2001.

29. V. Arakelian and M. Dahan. Complete shaking force and partial shaking moment balancing of planar four-bar linkages. Proceedings of the Institution of Mechanical Engineers, Part K: Journal of Multi-body Dynamics, 15:31-34, 2001.

30. S. Zhang. A constitutive method of objective function for the dynamic optimum balance of shaking force in linkage. Mechanism and Machine Theory, 29(6):829-835, 1994.

31. S. Zhang and J. Chen. The optimum balance of shaking force and shaking moment of linkages. Mechanism and Machine Theory, 30(4):589-597, 1995.

32. N.M. Qi and E. Pennestri. Optimum balancing of four-bar linkages. Mechanism and Machine Theory, 26(3):337-348, 1991.

33. H. Chaudhary and S.K. Saha. Balancing of four-bar linkages using maximum recursive dynamic algorithm. Mechanism and Machine Theory, 42(2):216-232, 2007.

34. V.A. Kamenski. On the question of the balancing of planar linkages. Mechanisms, 3(4):303322, 1968.

35. R.S. Berkof. Complete force and moment balancing of inline four-bar linkages. Mechanism and Machine Theory, 8(3):397-410, 1973.

36. C. Bagci. Complete shaking force and shaking moment balancing of link mechanisms using balancing idler loops. ASME Journal of Mechanical Design, 104:482-493, 1982.

37. Z. Ye and M.R. Smith. Complete balancing of planar linkages by an equivalence method. Mechanism and Machine Theory, 29(5):701-712, 1991.

38. V. Arakelian and M.R. Smith. Complete shaking force and shaking moment balancing of linkages. Mechanism and Machine Theory, 34(8):1141-1153, 1999.

39. F. Gao. Complete shaking force and shaking moment balancing of 17 types of eight-bar linkages only with revolute pairs. Mechanism and Machine Theory, 26(2):197-206, 1991.

40. L.B. Berestov. Comparative analysis of the reactions in the kinematic pairs of the four-bar linkages for the different methods of balancing. Journal of Mechanics of machines, pages 61-70, 1977. in Russian.

41. H. Dresig, S. Naake, and L. Rockausen. Vollständiger und harmonischer Ausgleich ebener Mechanismen. VDI Verlag, Düsseldorf, 1994.

42. I. Esat and H. Bahai. A theory of complete force and moment balancing of planar linkage mechanisms. Mechanism and Machine Theory, 34(6):903-922, 1999.

43. V. Arakelian and M. Dahan. Balanced four-bar articulated mechanism has output member extended beyond axis of second pivot and meshing gears, may 2002.

44. I.S. Kochev. Full shaking moment balancing of planar linkages by a prescribed input speed fluctuation. Mechanism and Machine Theory, 25(4):459-466, 1990. 
45. R. Ricard and C.M. Gosselin. On the development of reactionless parallel manipulators. In Proceedings of the ASME Design Engineering Technical Conferences and Computers and Information in Engineering Conference (IDETC/CIE 2000), Baltimore, Maryland, 2000.

46. Y. Wu and C.M. Gosselin. Synthesis of reactionless spatial 3-dof and 6-dof mechanisms without separate counter-rotations. The International Journal of Robotics Research, 23(6):625642, 2004.

47. C.M. Gosselin, F. Vollmer, G. Côté, and Y. Wu. Synthesis and design of reactionless threedegree-of-freedom parallel mechanisms. IEEE Transactions on Robotics and Automation, 20(2):191-199, 2004.

48. C.M. Gosselin, B. Moore, and J. Schicho. Dynamic balancing of planar mechanisms using toric geometry. Journal of Symbolic Computation, 44(9):1346-1358, 2009.

49. Q. Jiang and C.M. Gosselin. Dynamic optimization of reactionless 4-bar linkages. ASMEJournal of Dynamic System, Measurement and Control, 132, 2010.

50. B. Demeulenaere and R. Berkof. Improving machine drive dynamics: a structured design approach towards balancing. ASME Journal of Mechanical Design, 130(8), 2008.

51. R.S. Berkof. The input torque in linkages. Mechanism and Machine Theory, 14(1):61-73, 1979.

52. Y. Wu and C.M. Gosselin. On the dynamic balancing of multi-dof parallel mechanisms with multiple legs. ASME Journal of Mechanical Design, 129:234-238, 2007.

53. G.H. Martin. Kinematics and Dynamics of Machines. Columbus, OH: McGraw-Hill, 3rd edition, 2002.

54. S.K. Agrawal and A. Fattah. Reactionless space and ground robots: novel design and concept studies. Mechanism and Machine Theory, 39:25-40, 2004.

55. J. Wang and C.M. Gosselin. Static balancing of spatial three-degree-of-freedom parallel mechanisms. Mechanism and Machine Theory, 34:437-452, 1999.

56. W.S. Newman and N. Hogan. the optimal control of balanced manipulators. In Proceedings of the ASME winter annual meeting, CA, USA, 1986.

57. T. Laliberté, C.M. Gosselin, and M. Jean. Static balancing of 3-DOF planar parallel mechanisms. IEEE/ASME Transactions on Mechatronics, 4(4):363-377, 1999.

58. K. Fujikoshi. Balancing apparatus for jointed robot, oct 1976.

59. J. Wang and C.M. Gosselin. Static balancing of spatial four-degree-of-freedom parallel mechanisms. Mechanism and Machine Theory, 35(4):563-592, 2000.

60. A. Russo, R. Sinatra, and F. Xi. Static balancing of parallel robots. Mechanism and Machine Theory, 40(2):191-202, 2005.

61. P.R. Ouyang and W.J. Zhang. Force balancing of robotic mechanisms based on adjustment of kinematic parameters. ASME Journal of Mechanical Design, 127:433-440, 2005.

62. J.L. Herder and C.M. Gosselin. A counter-rotary counterweight for light-weight dynamic balancing. In Proceedings of the ASME Design Engineering Technical Conferences and Computers and Information in Engineering Conference (IDETC/CIE 2004), pages 659-667, Salt Lake City, Utah, USA, sep 2004.

63. A. Fattah and S.K. Agrawal. On the design of reactionless 3-dof planar parallel mechanisms. Mechanism and Machine Theory, 41(1):70-82, 2006.

64. S. Foucault and C.M. Gosselin. Synthesis, design, and prototyping of a planar three degreeof-freedom reactionless parallel mechanism. ASME Journal of Mechanical Design, 126:992999, 2004.

65. Y. Wu and C.M. Gosselin. Design of reactionless 3-dof and 6-dof parallel manipulators using parallelepiped mechanisms. IEEE Transactions on Robotics, 21(5):821-833, 2005.

66. E. Papadopoulos and A. Abu-Abed. Design and motion planning for a zero-reaction manipulator. In Proceedings of the IEEE International Conference on Robotics and Automation (ICRA 1994), pages 1554-1559, San Diego, CA, USA, 1994.

67. V. Arakelian and S. Briot. Dynamic balancing of the scara robot. In Proceedings of 17th CISMIFToMM Symposium on Robot Design, Dynamics, and Control (RoManSy 2008), Tokyo, Japan, 2008 . 
68. S. Briot and V. Arakelian. Complete shaking force and shaking moment balancing of the position-orientation decoupled PAMINSA manipulator. In Proceedings of the IEEE/ASME International Conference on Advanced Intelligent Mechatronics (AIM2009), Singapore, 2009.

69. S. Briot, V. Arakelian, and J.P. Le Baron. Shaking force minimization of high-speed robots via centre of mass acceleration control. Mechanism and Machine Theory, 57:1-12, 2012.

70. K.V. Frolov. Theory of mechanisms and machines. Vishaya shkola, 1987.

71. V.I. Doronin and A.I. Pospelov. Balanced slider-crank mechanism, 1991.

72. V. Arakelian. équilibrage dynamique complet des mécanismes. Mechanism and Machine Theory, 33(4):425-436, 1998.

73. C. Baradat, V. Arakelian, S. Briot, and S. Guegan. Design and prototyping of a new balancing mechanism for spatial parallel manipulators. ASME Journal of Mechanical Design, 130(7), 2008.

74. M. Leblond and C.M. Gosselin. Static balancing of spatial and planar parallel manipulators with prismatic actuators. In Proceedings of the ASME 1998 DETC Conference, 1998.

75. W. Seyferth. Massenersatz duch punktmassen in rumlichen getrieben. Mechanism and Machine Theory, 9:49-59, 1974. 